January 22, 2021

\title{
NILPOTENT ORBITS AND MIXED GRADINGS OF SEMISIMPLE LIE ALGEBRAS
}

\author{
DMITRI I. PANYUSHEV
}

\begin{abstract}
Let $\sigma$ be an involution of a complex semisimple Lie algebra $\mathfrak{g}$ and $\mathfrak{g}=\mathfrak{g}_{0} \oplus$ $\mathfrak{g}_{1}$ the related $\mathbb{Z}_{2}$-grading. We study relations between nilpotent $G_{0}$-orbits in $\mathfrak{g}_{0}$ and the respective $G$-orbits in $\mathfrak{g}$. If $e \in \mathfrak{g}_{0}$ is nilpotent and $\{e, h, f\} \subset \mathfrak{g}_{0}$ is an $\mathfrak{s l}_{2}$-triple, then the semisimple element $h$ yields a $\mathbb{Z}$-grading of $\mathfrak{g}$. Our main tool is the combined $\mathbb{Z} \times \mathbb{Z}_{2^{-}}$ grading of $\mathfrak{g}$, which is called a mixed grading. We prove, in particular, that if $e_{\sigma}$ is a regular nilpotent element of $\mathfrak{g}_{0}$, then the weighted Dynkin diagram of $e_{\sigma}, \mathcal{D}\left(e_{\sigma}\right)$, has only isolated zeros. It is also shown that if $G \cdot e_{\sigma} \cap \mathfrak{g}_{1} \neq \varnothing$, then the Satake diagram of $\sigma$ has only isolated black nodes and these black nodes occur among the zeros of $\mathcal{D}\left(e_{\sigma}\right)$. Using mixed gradings related to $e_{\sigma}$, we define an inner involution $\check{\sigma}$ such that $\sigma$ and $\check{\sigma}$ commute. Here we prove that the Satake diagrams for both $\check{\sigma}$ and $\sigma \check{\sigma}$ have isolated black nodes.
\end{abstract}

\section{INTRODUCTION}

Let $G$ be a complex semisimple algebraic group with Lie $G=\mathfrak{g}, \operatorname{lnv}(\mathfrak{g})$ the set of involutions of $\mathfrak{g}$, and $\mathcal{N} \subset \mathfrak{g}$ the set of nilpotent elements. If $\sigma \in \operatorname{lnv}(\mathfrak{g})$, then $\mathfrak{g}=\mathfrak{g}_{0} \oplus \mathfrak{g}_{1}$ is the corresponding $\mathbb{Z}_{2}$-grading, i.e., $\mathfrak{g}_{i}=\mathfrak{g}_{i}^{(\sigma)}$ is the $(-1)^{i}$-eigenspace of $\sigma$. Let $G_{0}$ denote the connected subgroup of $G$ with Lie $G_{0}=\mathfrak{g}_{0}$. Study of the (nilpotent) $G_{0}$-orbits in $\mathfrak{g}_{1}$ is closely related to the study of the (nilpotent) $G$-orbits in $\mathfrak{g}$ meeting $\mathfrak{g}_{1}$. The corresponding theory has been developed in [10]. In this article, we look at the $\mathbb{Z}_{2}$-graded situation from another angle and study properties of nilpotent $G$-orbits meeting $\mathfrak{g}_{0}$. Roughly speaking, the general problem is two-fold:

(a) describe the $G$-orbits in $\mathcal{N}$ that meet $\mathfrak{g}_{0}$ and then the $G_{0}$-orbits in $G \cdot e \cap \mathfrak{g}_{0}$;

(b) given an orbit $G_{0} \cdot e \subset \mathfrak{g}_{0} \cap \mathcal{N}$, determine the (properties of) orbit $G \cdot e$.

The well-known classification of the nilpotent orbits in $\mathfrak{s o}_{n}$ or $\mathfrak{s p}_{2 n}$ via partitions comprise a solution to (a) for the pairs $\left(\mathfrak{g}, \mathfrak{g}_{0}\right)=\left(\mathfrak{s l}_{n}, \mathfrak{s o}_{n}\right)$ or $\left(\mathfrak{s l}_{2 n}, \mathfrak{s p}_{2 n}\right)$, i.e., for the outer involutions of $\mathfrak{s l}_{n}$. Here we provide some general results related to (b) and their applications.

For $e \in \mathfrak{g}_{0} \cap \mathcal{N}$, an $\mathfrak{s l}_{2}$-triple containing $e$, say $\{e, h, f\}$, can be chosen in $\mathfrak{g}_{0}$. Combining the $\mathbb{Z}_{2}$-grading $\mathfrak{g}=\mathfrak{g}_{0} \oplus \mathfrak{g}_{1}$ and $\mathbb{Z}$-grading $\mathfrak{g}=\bigoplus_{i \in \mathbb{Z}} \mathfrak{g}(i)$ determined by $h$ yields a $\mathbb{Z} \times \mathbb{Z}_{2^{-}}$ grading $\mathfrak{g}=\bigoplus_{(i, j) \in \mathbb{Z} \times \mathbb{Z}_{2}} \mathfrak{g}_{j}(i)$, which is called a mixed grading related to $(\sigma, e)$ or just a $(\sigma, e)$-grading. Here $e \in \mathfrak{g}_{0}(2)$ and $h \in \mathfrak{g}_{0}(0)$. Let $e_{\sigma}$ denote a regular nilpotent element of

2010 Mathematics Subject Classification. 17B08, 17B70, 14L30.

Key words and phrases. Centraliser, weighted Dynkin diagram, grading, involution.

This research was funded by RFBR, project № 20-01-00515. 
$\mathfrak{g}_{0}=\mathfrak{g}_{0}^{(\sigma)}$. We prove that the weighted Dynkin diagram of $G \cdot e_{\sigma}, \mathcal{D}\left(e_{\sigma}\right)$, has only isolated zeros. Moreover, if $G \cdot e_{\sigma} \cap \mathfrak{g}_{1}^{\left(\sigma^{\prime}\right)} \neq \varnothing$ for some $\sigma^{\prime} \in \operatorname{lnv}(\mathfrak{g})$, then the Satake diagram of $\sigma^{\prime}$, denoted Sat $\left(\sigma^{\prime}\right)$, has only isolated black nodes (IBN for short), and these black nodes occur among the zeros of $\mathcal{D}\left(e_{\sigma}\right)$. Let $c(\mathfrak{g})$ be the Coxeter number of a simple Lie algebra $\mathfrak{g}$. We show the orbit $G \cdot e_{\sigma} \subset \mathcal{N}$ is even whenever $\mathrm{c}(\mathfrak{g})$ is even. It is also proved that if $\mathfrak{g}_{0}$ is semisimple and $e$ is distinguished in $\mathfrak{g}_{0}$, then $e$ remains even in $\mathfrak{g}$ and the reductive part of the centraliser $\mathfrak{g}^{e}$ is toral (i.e., $e$ is almost distinguished in $\mathfrak{g}$ ), see Section 3.

For a simple Lie algebra $\mathfrak{g}$, let $\kappa(\mathfrak{g})$ denote the maximal number of pairwise disjoint nodes in the Dynkin diagram. If $\mathrm{c}(\mathfrak{g})$ is even (i.e., $\mathfrak{g} \neq \mathfrak{s l}_{2 n+1}$ ), then we prove that there is a unique, up to $G$-conjugation, inner involution $\vartheta$ such that $\mathcal{D}\left(e_{\vartheta}\right)$ has $\kappa(\mathfrak{g})$ isolated zeros. This $\vartheta$ is characterised by the property that $\mathfrak{g}_{1}^{(\vartheta)}$ contains a regular nilpotent element of $\mathfrak{g}$. Let $B$ be a Borel subgroup of $G$ and $U=(B, B)$. Using $\vartheta$, we also show that $B$ has a dense orbit in $\mathfrak{u}^{\prime}=[\mathfrak{u}, \mathfrak{u}]$, where $\mathfrak{u}=\operatorname{Lie} U$, and if $\mathcal{O}$ is the dense $B$-orbit in $\mathfrak{u}^{\prime}$, then $G \cdot \mathcal{O}=G \cdot e_{\vartheta}$, see Section 4.

As in [14], we say that the orbit $G \cdot e \subset \mathcal{N}$ is divisible, if $\frac{1}{2} \mathcal{D}(e)$ is again a weighted Dynkin diagram. Then the respective nilpotent orbit is denoted by $G \cdot e^{\langle 2\rangle}$. There is an interesting link between mixed gradings and divisible $G$-orbits. If a $(\sigma, e)$-grading has the property that $\operatorname{dim} \mathfrak{g}_{0}(0)=\operatorname{dim} \mathfrak{g}_{1}(4)$, then we prove that $G \cdot e$ is divisible, $\mathfrak{g}_{0}$ is semisimple, and $\operatorname{dim} \mathfrak{g}_{0}(4 k+2)=\operatorname{dim} \mathfrak{g}_{1}(4 k+2)$ for all $k \in \mathbb{Z}$. Moreover, both $e$ and $e^{\langle 2\rangle}$ are almost distinguished in $\mathfrak{g}$ and all such instances are classified, see Section 5 for details.

Given $\sigma \in \operatorname{lnv}(\mathfrak{g})$, we define $\Upsilon(\sigma) \in \operatorname{Inv}(\mathfrak{g})$, if $\sigma$ is not an inner involution of $\mathfrak{s l}_{2 n+1}$. Constructing the map $\Upsilon$ depends on a choice of $e_{\sigma} \in \mathfrak{g}_{0}^{(\sigma)} \cap \mathcal{N}$ and exploits the mixed grading related to $\left(\sigma, e_{\sigma}\right)$, see Section 6. Here $\check{\sigma}:=\Upsilon(\sigma)$ is always inner, and the involutions $\sigma$ and $\check{\sigma}$ commute. Hence $\sigma \check{\sigma} \in \operatorname{lnv}(\mathfrak{g})$ and $\sigma, \sigma \check{\sigma}$ belong to the same connected component of the group Aut $(\mathfrak{g})$. The map $\Upsilon$ has the property that $e_{\sigma} \in \mathfrak{g}_{1}^{(\check{\sigma})}$ and $e_{\sigma} \in \mathfrak{g}_{1}^{(\sigma \check{\sigma})}$. This implies that the Satake diagrams Sat $(\check{\sigma})$ and Sat $(\sigma \check{\sigma})$ have only IBN. We describe a method that allows us to compute (the conjugacy class of) $\check{\sigma}$ or $\sigma \check{\sigma}$. We also discuss the property that $\sigma$ and $\sigma \check{\sigma}$ are $G$-conjugate, and its connection to the divisibility of $G \cdot e_{\sigma}$. The commuting involutions $\sigma$ and $\check{\sigma}$ provide a $\mathbb{Z}_{2} \times \mathbb{Z}_{2}$-grading of $\mathfrak{g}$, and our construction based on a mixed grading yields an explicit model for it.

Main notation. Let $\mathfrak{h}$ be a fixed Cartan subalgebra of $\mathfrak{g}, \Delta$ the root system of $(\mathfrak{g}, \mathfrak{h})$, and $\Pi$ a set of simple roots. If $\gamma \in \Delta$, then $\mathfrak{g}_{\gamma}$ is the root space in $\mathfrak{g}$. Write $\mathfrak{g}^{x}$ or $\mathfrak{z}_{\mathfrak{g}}(x)$ for the centraliser of $x \in \mathfrak{g}$ in $\mathfrak{g}$. More generally, $\mathfrak{z}_{\mathfrak{g}}(M)=\cap_{x \in M \mathfrak{z}_{\mathfrak{g}}}(x)$ for a subset $M$ of $\mathfrak{g}$. If $G \cdot e \subset \mathcal{N}$ is a nonzero orbit, then $\mathcal{D}(e)$ is its weighted Dynkin diagram. A direct sum of Lie algebras is denoted by ' $\dot{+}$ '.

Our man reference for algebraic groups and Lie algebras is [24]. We refer to [3] for generalities on nilpotent elements (orbits) and their centralisers. 


\section{PRELIMINARIES ON NILPOTENT ORBITS AND INVOLUTIONS}

Suppose for a while that $\mathfrak{g}$ is a reductive algebraic Lie algebra. We say that $x \in \mathfrak{g}$ is regular, if $\operatorname{dim} \mathfrak{g}^{x}=\operatorname{rk}(\mathfrak{g})$. The set of regular elements is denoted by $\mathfrak{g}_{\text {reg }}$. Let $\mathcal{N}$ be the set of nilpotent elements of $\mathfrak{g}$. For $e \in \mathcal{N} \backslash\{0\}$, let $\{e, h, f\}$ be an $\mathfrak{s l}_{2}$-triple in $\mathfrak{g}$. That is, $[h, e]=2 e,[e, f]=h$, and $[h, f]=-2 f$. The semisimple element $h$ is called a characteristic of e. W.l.o.g. one may assume that $h \in \mathfrak{h}$ and $\alpha(h) \geqslant 0$ for all $\alpha \in \Pi$. By a celebrated result of Dynkin, one then has $\alpha(h) \in\{0,1,2\}$ [4, Theorem 8.3]. The weighted Dynkin diagram of $G \cdot e(=$ of $e), \mathcal{D}(e)$, is the Dynkin diagram of $\mathfrak{g}$ equipped with labels $\{\alpha(h)\}_{\alpha \in \Pi \text {. The set of }}$ zeros of $\mathcal{D}(e)$ is the subset $\Pi_{0}=\{\alpha \in \Pi \mid \alpha(h)=0\}$.

Let $\mathfrak{g}=\bigoplus_{i \in \mathbb{Z}} \mathfrak{g}(i)$ be the $\mathbb{Z}$-grading determined by $h$, i.e., $\mathfrak{g}(i)=\{v \in \mathfrak{g} \mid[h, v]=i v\}$. Then $\mathfrak{g}^{e}$ inherits this $\mathbb{Z}$-grading and $\mathfrak{g}(0)=\mathfrak{g}^{h}$. Recall some standard definitions related to nilpotent elements and $\mathfrak{s l}_{2}$-triples. A nonzero $e \in \mathcal{N}$ is said to be

- even, if the $h$-eigenvalues in $\mathfrak{g}$ are even;

- distinguished, if $\mathfrak{z}_{\mathfrak{g}}(e, h, f)$ is the centre of $\mathfrak{g}$ (i.e., $\mathfrak{z}_{\mathfrak{g}}(e, h, f)=0$, if $\mathfrak{g}$ is semisimple);

- almost distinguished, if $\mathfrak{z}_{\mathfrak{g}}(e, h, f)$ is a toral Lie algebra (= Lie algebra of a torus);

Write $\mathfrak{t}_{n}$ for an $n$-dimensional toral Lie algebra. For $e \in \mathfrak{g}_{\text {reg }} \cap \mathcal{N}$, any $\mathfrak{s l}_{2}$-triple $\{e, h, f\}$ is said to be principal (in $\mathfrak{g}$ ). Set $\mathfrak{g}(\geqslant j)=\bigoplus_{i \geqslant j} \mathfrak{g}(i)$. It is well known that

(i) ad $e: \mathfrak{g}(i) \rightarrow \mathfrak{g}(i+2)$ is injective (resp. surjective) if $i \leqslant-1$ (resp. $i \geqslant-1$ ). Hence $\mathfrak{g}^{e} \subset \mathfrak{g}(\geqslant 0), \operatorname{dim} \mathfrak{g}^{e}=\operatorname{dim} \mathfrak{g}(0)+\operatorname{dim} \mathfrak{g}(1)=\operatorname{dim} \mathfrak{g}^{h}+\operatorname{dim} \mathfrak{g}(1)$, and $e$ is even if and only if $\operatorname{dim} \mathfrak{g}^{e}=\operatorname{dim} \mathfrak{g}^{h}$. Furthermore, $(\operatorname{ad} e)^{i}: \mathfrak{g}(-i) \rightarrow \mathfrak{g}(i)$ is bijective.

(ii) a regular element is distinguished and a distinguished element is even [3, Theorem 8.2.3]; however, there exist almost distinguished non-even elements.

(iii) $\mathfrak{z}_{\mathfrak{g}}(e, h, f)=\mathfrak{g}^{e}(0)$ is a maximal reductive subalgebra of $\mathfrak{g}^{e}[3,3.7]$. We also write $\mathfrak{g}_{\text {red }}^{e}$ for it. Then $\operatorname{dim} \mathfrak{g}_{\text {red }}^{e}=\operatorname{dim} \mathfrak{g}(0)-\operatorname{dim} \mathfrak{g}(2)$ and $e$ is distinguished (resp. almost distinguished) if and only if $\mathfrak{g}^{e}$ has no non-central semisimple elements (resp. is solvable).

(iv) The nilradical of $\mathfrak{g}^{e}, \mathfrak{g}_{\text {nil }}^{e}$, is contained in $\mathfrak{g}(\geqslant 1)$ and $\operatorname{dim} \mathfrak{g}_{\text {nil }}^{e}=\operatorname{dim} \mathfrak{g}(1)+\operatorname{dim} \mathfrak{g}(2)$.

From now on, $\mathfrak{g}$ is assumed to be semisimple. Let $\sigma$ be an involution of $\mathfrak{g}$ and $\mathfrak{g}=\mathfrak{g}_{0} \oplus \mathfrak{g}_{1}$ the corresponding $\mathbb{Z}_{2}$-grading. Then $\mathfrak{g}_{0}=\mathfrak{g}^{\sigma}$ is reductive, but not necessarily semisimple. We also say that $\left(\mathfrak{g}, \mathfrak{g}_{0}\right)$ is a symmetric pair. Whenever we wish to stress that $\mathfrak{g}_{i}$ is defined via certain $\sigma \in \operatorname{lnv}(\mathfrak{g})$, especially when several involutions are being considered simultaneously, we write $\mathfrak{g}_{i}^{(\sigma)}$ for it. We can also write $\mathfrak{g}^{\sigma}$ in place of $\mathfrak{g}_{0}^{(\sigma)}$.

The centraliser $\mathfrak{g}^{x}$ is $\sigma$-stable for any $x \in \mathfrak{g}_{i}$, hence $\mathfrak{g}^{x}=\mathfrak{g}_{0}^{x} \oplus \mathfrak{g}_{1}^{x}$. It is known that

- if $x \in \mathfrak{g}_{1}$, then $\operatorname{dim} G_{0} \cdot x=\frac{1}{2} \operatorname{dim} G \cdot x$; that is, $\operatorname{dim} \mathfrak{g}_{1}-\operatorname{dim} \mathfrak{g}_{1}^{x}=\operatorname{dim} \mathfrak{g}_{0}-\operatorname{dim} \mathfrak{g}_{0}^{x}$ [10, Proposition 5].

- if $0 \neq x \in \mathfrak{g}_{0}$, then $\operatorname{dim} \mathfrak{g}_{0}^{x}+\mathrm{rk}(\mathfrak{g})>\operatorname{dim} \mathfrak{g}_{1}^{x}[16$, Theorem 4.4].

It is well known that the real forms of $\mathfrak{g}$ are represented by their Satake diagrams (see 
e.g. [24, Ch. 4,§4.3]) and there is a one-to-one correspondence between the real forms and $\mathbb{Z}_{2}$-gradings of $\mathfrak{g}$. Thereby, one associates the Satake diagram to an involution (symmetric pair), cf. [20]. A Satake diagram of $\sigma$, Sat $(\sigma)$, is the Dynkin diagram of $\mathfrak{g}$, with black and white nodes, where certain pairs of white nodes can be joined by an arrow. Let $x \in \mathfrak{g}_{1}$ be a generic semisimple element. Then $\mathfrak{g}_{1}^{x}$ is a toral subalgebra (a Cartan subspace of $\mathfrak{g}_{1}$ ) and $\operatorname{Sat}(\sigma)$ encodes the structure of $\mathfrak{g}_{0}^{x}$. In particular, the subdiagram of black nodes in Sat $(\sigma)$ represents the Dynkin diagram of $\left[\mathfrak{g}^{x}, \mathfrak{g}^{x}\right]=\left[\mathfrak{g}_{0}^{x}, \mathfrak{g}_{0}^{x}\right]$, while the number of arrows equals $\operatorname{dim}\left(\mathfrak{g}_{0}^{x} /\left[\mathfrak{g}_{0}^{x}, \mathfrak{g}_{0}^{x}\right]\right)$. Some features of Satake diagrams in the setting of $\mathbb{Z}_{2}$-gradings are discussed in [17, Sect. 2].

Example 2.1. As $G / G_{0}$ is a spherical homogeneous space, $\operatorname{dim} \mathfrak{g}_{1}=\operatorname{dim}\left(G / G_{0}\right) \leqslant \operatorname{dim} B$. Hence $\operatorname{dim} \mathfrak{g}_{0} \geqslant \operatorname{dim} U$ and $\operatorname{dim} \mathfrak{g}_{1}-\operatorname{dim} \mathfrak{g}_{0} \leqslant \operatorname{rk}(\mathfrak{g})$ for any $\sigma \in \operatorname{lnv}(\mathfrak{g})$. If $\operatorname{dim} \mathfrak{g}_{1}-\operatorname{dim} \mathfrak{g}_{0}=$ $\mathrm{rk}(\mathfrak{g})$, then $\sigma$ is said to be of maximal rank. (In [20], such involutions are called split.) Equivalently, $\mathfrak{g}_{1}$ contains a Cartan subalgebra of $\mathfrak{g}$. For any simple $\mathfrak{g}$, there is a unique, up to $G$-conjugacy, involution of maximal rank, and we denote it by $\vartheta_{\max }$. In this case, $\mathfrak{g}^{x} \cap \mathfrak{g}_{0}^{\left(\vartheta_{\max }\right)}=\{0\}$ for a generic $x \in \mathfrak{g}_{1}^{\left(\vartheta_{\max }\right)}$. Hence $\operatorname{Sat}\left(\vartheta_{\text {max }}\right)$ has neither black nodes nor arrows. Yet another characterisation is that $\vartheta_{\max }$ corresponds to a split real form of $\mathfrak{g}$, see $[24$, Ch. $4, \S 4.4]$.

Remark 2.2. By a fundamental result of Antonyan, for any $\sigma \in \operatorname{lnv}(\mathfrak{g})$ and an $\mathfrak{s l}_{2}$-triple $\{e, h, f\} \subset \mathfrak{g}$, one has $G \cdot e \cap \mathfrak{g}_{1} \neq \varnothing$ if and only if $G \cdot h \cap \mathfrak{g}_{1} \neq \varnothing$, see [1, Theorem 1]. This readily implies that $\sigma=\vartheta_{\max }$ if and only if $G \cdot x \cap \mathfrak{g}_{1} \neq \varnothing$ for any $x \in \mathfrak{g}$ ([1, Theorem 2]). For arbitrary $\sigma$ and $e \in \mathcal{N}$, this means that $G \cdot e \cap \mathfrak{g}_{1} \neq \varnothing$ if and only if (i) the set of black nodes of $\operatorname{Sat}(\sigma)$ is contained in the set of zeros of $\mathcal{D}(e)$ and (ii) $\alpha(h)=\beta(h)$ whenever the nodes $\alpha, \beta \in \Pi$ are joined by an arrow in $\operatorname{Sat}(\sigma)$.

\section{MiXed GRADINGS OF SEMISIMPLE LIE ALGEBRAS}

A mixed grading of $\mathfrak{g}$ is a grading via the group $\mathbb{Z} \times \mathbb{Z}_{2}$. We consider only mixed gradings of a special form. Given $\sigma \in \operatorname{lnv}(\mathfrak{g})$ and a nonzero $e \in \mathfrak{g}_{0} \cap \mathcal{N}$, take an $\mathfrak{s l}_{2}$-triple $\{e, h, f\}$ in $\mathfrak{g}_{0}$. Then we set $\mathfrak{g}_{j}(i)=\left\{v \in \mathfrak{g}_{j} \mid[h, v]=i v\right\}$ and consider the mixed grading

$$
\mathfrak{g}=\bigoplus_{j \in \mathbb{Z}_{2}} \bigoplus_{i \in \mathbb{Z}} \mathfrak{g}_{j}(i)=\bigoplus_{(i, j) \in \mathbb{Z} \times \mathbb{Z}_{2}} \mathfrak{g}_{j}(i)
$$

We say that (3.1) is a mixed grading related to $(\sigma, e)$ or just a $(\sigma, e)$-grading. Here $e \in \mathfrak{g}_{0}(2)$ and $f \in \mathfrak{g}_{0}(-2)$. For such a mixed grading, it follows from (i) in Section 2 that

$$
\left\{\begin{array}{l}
\operatorname{ad} e: \mathfrak{g}_{j}(i) \rightarrow \mathfrak{g}_{j}(i+2) \text { is surjective for } j=0,1 \text { and } i \geqslant-1 . \\
\text { ad } e: \mathfrak{g}_{j}(i) \rightarrow \mathfrak{g}_{j}(i+2) \text { is injective for } j=0,1 \text { and } i \leqslant-1 .
\end{array}\right.
$$


Letting $d_{j}(i)=\operatorname{dim} \mathfrak{g}_{j}(i)$, we have $d_{j}(i) \geqslant d_{j}(i+2)$ for $i \geqslant-1$ and $d_{j}(i)=d_{j}(-i)$. Below, we mostly consider even nilpotent elements of $\mathfrak{g}_{0}$. However, if $e \in \mathfrak{g}_{0}$ is even in $\mathfrak{g}_{0}$, then $e$ is not necessarily even in $\mathfrak{g}$.

Lemma 3.1. If $\mathfrak{g}$ is simple and $e$ is even in $\mathfrak{g}_{0}$, then the $h$-eigenvalues in $\mathfrak{g}_{1}$ are either all even or all odd.

Proof. Write $\mathfrak{g}_{1}=\mathfrak{g}_{1}^{+} \oplus \mathfrak{g}_{1}^{-}$, where the $h$-eigenvalues in $\mathfrak{g}_{1}^{+}$(resp. $\mathfrak{g}_{1}^{-}$) are even (resp. odd). Since the $h$-eigenvalues in $\mathfrak{g}_{0}$ are even, we have $\left[\mathfrak{g}_{0}, \mathfrak{g}_{1}^{ \pm}\right] \subset \mathfrak{g}_{1}^{ \pm} ;$also $\left[\mathfrak{g}_{1}^{+}, \mathfrak{g}_{1}^{-}\right] \subset \mathfrak{g}_{0}$. On the other hand, the $h$-eigenvalues in $\left[\mathfrak{g}_{1}^{+}, \mathfrak{g}_{1}^{-}\right]$are odd. This implies that $\left[\mathfrak{g}_{1}^{+}, \mathfrak{g}_{1}^{-}\right]=0$. Therefore, letting $\tilde{\mathfrak{g}}_{0}=\mathfrak{g}_{0} \oplus \mathfrak{g}_{1}^{+}$and $\tilde{\mathfrak{g}}_{1}=\mathfrak{g}_{1}^{-}$, we obtain another $\mathbb{Z}_{2}$-grading of $\mathfrak{g}$. As is well known, for a symmetric pair $\left(\mathfrak{g}, \mathfrak{g}_{0}\right)$ with simple Lie algebra $\mathfrak{g}$, $\mathfrak{g}_{0}$ is a maximal proper reductive subalgebra. Consequently, either $\tilde{\mathfrak{g}}_{0}=\mathfrak{g}_{0}$ or $\tilde{\mathfrak{g}}_{0}=\mathfrak{g}$.

Lemma 3.2. Suppose that $\mathfrak{g}_{0}$ is semisimple and $e \in \mathfrak{g}_{0} \cap \mathcal{N}$ is distinguished in $\mathfrak{g}_{0}$. Then $e$ is almost distinguished and even in $\mathfrak{g}$.

Proof. The assumptions imply that $\mathfrak{z}_{\mathfrak{g}}(e, h, f) \cap \mathfrak{g}_{0}=\{0\}$. Therefore, $\mathfrak{z}_{\mathfrak{g}}(e, h, f) \subset \mathfrak{g}_{1}$. Then $\mathfrak{z}_{\mathfrak{g}}(e, h, f)$ is reductive and abelian, hence toral.

Since $e$ is even in $\mathfrak{g}_{0}$, it follows from Lemma 3.1 that either $\mathfrak{g}_{1}=\mathfrak{g}_{1}^{+}$or $\mathfrak{g}_{1}=\mathfrak{g}_{1}^{-}$. Assume that $\mathfrak{g}_{1}=\mathfrak{g}_{1}^{-}$. Since $\left(\mathfrak{g}_{1}^{-}\right)^{h}=\{0\}$, we obtain $\mathfrak{z}_{\mathfrak{g}}(e, h, f)=\mathfrak{g}_{\mathfrak{g} 0}(e, h, f)=0$. Therefore, $e$ is distinguished in $\mathfrak{g}$ and hence even. This contradicts the fact that $\mathfrak{g}_{1}^{-}$is nontrivial. Thus, $\mathfrak{g}_{1}=\mathfrak{g}_{1}^{+}$and $e$ is even in $\mathfrak{g}$, although not necessarily distinguished.

For $\mathfrak{g} \in\{\mathfrak{s l}(\mathbb{V}), \mathfrak{s o}(\mathbb{V}), \mathfrak{s p}(\mathbb{V})\}$, the nilpotent orbits are represented by partitions of $\operatorname{dim} \mathbb{V}$, see [3, Ch. 5]. There are also simple algorithms for obtaining $\mathcal{D}(e)$ via $\boldsymbol{\lambda}=\boldsymbol{\lambda}(e)=\boldsymbol{\lambda}(G \cdot e)$, which go back to Springer and Steinberg [21, IV.4], and here $G \cdot e \subset \mathfrak{g}$ is even if and only if all parts of $\boldsymbol{\lambda}(e)$ have the same parity.

Example 3.3. a) If $\sigma \in \operatorname{lnv}\left(\mathfrak{s l}_{2 n+1}\right)$ is inner, then $\mathfrak{g}_{0}=\mathfrak{s l}_{k} \dot{+} \mathfrak{s l}_{2 n+1-k} \dot{+} \mathfrak{t}_{1}$ with $k<2 n+1-k$. If $e \in \mathfrak{g}_{0, \text { reg }}$, then $\boldsymbol{\lambda}(e)=(2 n+1-k, k)$ and $e$ is not even in $\mathfrak{g}$. Here $\mathfrak{g}_{0}$ is not semisimple and $\mathfrak{g}_{1}=\mathfrak{g}_{1}^{-}$.

b) Another example is $\mathfrak{g}=\mathfrak{s p}_{2 n}$ and $\mathfrak{g}_{0}=\mathfrak{s p}_{2 k} \dot{+} \mathfrak{s p}_{2 n-2 k}$ with $0<k<n$. If $e$ is regular in $\mathfrak{s p}_{2 k}$, then $\boldsymbol{\lambda}(G \cdot e)=(2 k, 1, \ldots, 1)$, which means that $e$ is not even in $\mathfrak{g}$.

c) It can happen that $\mathfrak{g}_{0}$ is simple and $e \in \mathfrak{g}_{0}$ is even, but $e$ is not even in $\mathfrak{g}$. For instance, take $\sigma \in \operatorname{Inv}\left(\mathbf{F}_{4}\right)$ such that $\left(\mathbf{F}_{4}\right)^{\sigma}=\mathfrak{s o}_{9}$. Let $e \in \mathfrak{s o}_{9}$ be such that $\boldsymbol{\lambda}(e)=(3,3,3)$. Then $\mathcal{D}(e)$ is (1)-(0)-2). Here (and in Tables 1,2 below) the shaded nodes in the weighted Dynkin diagrams represent the short simple roots.

Let $G(0)$ (resp. $G_{0}(0)$ ) be the connected subgroup of $G$ with Lie algebra $\mathfrak{g}(0)$ (resp $\mathfrak{g}_{0}(0)$ ).

Lemma 3.4. For any mixed grading of $\mathfrak{g}, G_{0}(0)$ has finitely many orbits in any $\mathfrak{g}_{j}(i)$ if $i \neq 0$. Consequently, for $i \neq 0$, there is a dense $G_{0}(0)$-orbit in $\mathfrak{g}_{j}(i)$ and hence $d_{j}(i) \leqslant d_{0}(0)$. 
Proof. The presence of the grading shows that $[\mathfrak{g}, x] \cap \mathfrak{g}_{j}(i)=\left[\mathfrak{g}_{0}(0), x\right]$ for any $x \in \operatorname{dim} \mathfrak{g}_{j}(i)$. By Vinberg's lemma [22, §2], this implies that the intersection of any $G$-orbit with $\mathfrak{g}_{j}(i)$ consists of finitely many $G_{0}(0)$-orbits. For $i \neq 0$, all elements of $\mathfrak{g}_{j}(i)$ are nilpotent. Therefore, there are finitely many (nilpotent) $G$-orbits meeting $\mathfrak{g}_{j}(i)$ with $i \neq 0$.

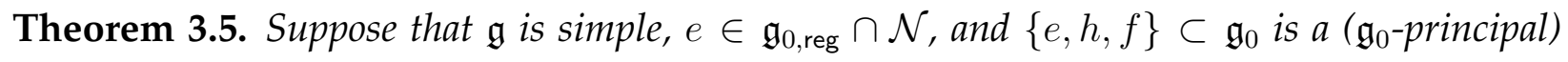
$\mathfrak{s l}_{2}$-triple. Then

(1) $\left[\mathfrak{g}^{h}, \mathfrak{g}^{h}\right] \simeq \mathfrak{s l}_{2} \dot{+} \cdots \dot{+} \mathfrak{s l}_{2}=\left(\mathfrak{s l}_{2}\right)^{k}$ for some $k \geqslant 0$ and $\mathcal{D}(e)$ has only isolated zeros. More precisely, here $\operatorname{dim} \mathfrak{g}^{h}=\mathrm{rk}(\mathfrak{g})+2 k$ and $\mathcal{D}(e)$ contains exactly $k$ isolated zeros;

(2) $\operatorname{dim} \mathfrak{g}_{\mathrm{nil}}^{e} \leqslant 2 \mathrm{rk}\left(\mathfrak{g}_{0}\right)$, and if the equality holds, then $\mathfrak{g}_{0}$ is semisimple.

Proof. Consider a mixed grading related to $(\sigma, e)$.

(1) The centraliser of $h$ in $\mathfrak{g}$ is reductive and $\mathbb{Z}_{2}$-graded: $\mathfrak{g}^{h}=\mathfrak{g}_{0}(0) \oplus \mathfrak{g}_{1}(0)$. Since $e$ is regular nilpotent in $\mathfrak{g}_{0}, \mathfrak{g}_{0}(0)$ is a Cartan subalgebra of $\mathfrak{g}_{0}$, and therefore $\left[\mathfrak{g}^{h}, \mathfrak{g}^{h}\right]_{0}$ is commutative. For a semisimple Lie algebra $\left[\mathfrak{g}^{h}, \mathfrak{g}^{h}\right]$, this is only possible if all its simple factors are isomorphic to $\mathfrak{s l}_{2}$. Each simple factor $\mathfrak{s l}_{2}$ of $\mathfrak{g}^{h}$ corresponds to a simple root, and the corresponding set of $k$ simple roots gives rise to a totally disconnected subset of the Dynkin diagram. Recall that the set of zeros of $\mathcal{D}(e)$ represent the Dynkin diagram of $\left[\mathfrak{g}^{h}, \mathfrak{g}^{h}\right]$. The rest is clear.

(2) Recall that $\operatorname{dim} \mathfrak{g}_{\text {nil }}^{e}=\operatorname{dim} \mathfrak{g}(1)+\operatorname{dim} \mathfrak{g}(2)$. By Lemma 3.1, either $\mathfrak{g}_{1}=\mathfrak{g}_{1}^{+}$or $\mathfrak{g}_{1}=\mathfrak{g}_{1}^{-}$. If $\mathfrak{g}_{1}=\mathfrak{g}_{1}^{+}$, then $\mathfrak{g}(1)=0$, and $\operatorname{dim} \mathfrak{g}_{\text {nil }}^{e}=\operatorname{dim} \mathfrak{g}_{0}(2)+\operatorname{dim} \mathfrak{g}_{1}(2) \leqslant 2 \operatorname{dim} \mathfrak{g}_{0}(0)=2 \mathrm{rk}\left(\mathfrak{g}_{0}\right)$. If $\mathfrak{g}_{1}=\mathfrak{g}_{1}^{-}$, then $\mathfrak{g}(1)=\mathfrak{g}_{1}(1)$ and $\mathfrak{g}(2)=\mathfrak{g}_{0}(2)$, with the similar estimate.

In both cases, if $\operatorname{dim} \mathfrak{g}_{\text {nil }}^{e}=2 \operatorname{rk}\left(\mathfrak{g}_{0}\right)$, then $\operatorname{dim} \mathfrak{g}_{0}(2)=\operatorname{dim} \mathfrak{g}_{0}(0)$. Since $\{e, h, f\} \subset\left[\mathfrak{g}_{0}, \mathfrak{g}_{0}\right]$ and $\mathfrak{g}_{0}(i) \subset\left[\mathfrak{g}_{0}, \mathfrak{g}_{0}\right]$ for $i \neq 0$, we have

$$
\operatorname{dim} \mathfrak{g}_{0}(2)=\operatorname{dim}\left[\mathfrak{g}_{0}, \mathfrak{g}_{0}\right](2) \leqslant \operatorname{dim}\left[\mathfrak{g}_{0}, \mathfrak{g}_{0}\right](0) \leqslant \operatorname{dim} \mathfrak{g}_{0}(0) .
$$

Hence $\left[\mathfrak{g}_{0}, \mathfrak{g}_{0}\right](0)=\mathfrak{g}_{0}(0)$ and therefore $\left[\mathfrak{g}_{0}, \mathfrak{g}_{0}\right]=\mathfrak{g}_{0}$, i.e., $\mathfrak{g}_{0}$ is semisimple.

Remark. It is proved by Broer [2] that if $e$ is even, $\mathcal{D}(e)$ has isolated zeros, and the set of zeros $\Pi_{0}$ consists of short roots, then the closure of $G \cdot e$ is normal. (If $\mathfrak{g}$ is simply-laced, then all roots are assumed to be short.)

In Theorem 3.5, $e \in \mathfrak{g}_{0, \text { reg }}$ is not necessarily even in $\mathfrak{g}$, and we characterise below possible exceptions. Write $\mathrm{c}(\mathfrak{g})$ for the Coxeter number of $\mathfrak{g}$. Let $\Delta^{+}$be the set of positive roots corresponding to $\Pi$ and $\theta \in \Delta^{+}$the highest root. For $\gamma \in \Delta$ and $\alpha \in \Pi$, let $[\gamma: \alpha]$ be the coefficient of $\alpha$ in the expression of $\gamma$ via $\Pi$. Then ht $(\gamma):=\sum_{\alpha \in \Pi}[\gamma: \alpha]$ and $\mathrm{c}(\mathfrak{g})=\mathrm{ht}(\theta)+1$.

Proposition 3.6. Suppose that $\mathfrak{g}$ is simple and $e \in \mathfrak{g}_{0, \text { reg }} \cap \mathcal{N}$. If $\mathrm{c}(\mathfrak{g})$ is even, then e is even in $\mathfrak{g}$. Moreover, $e$ is not even in $\mathfrak{g}$ if and only if $\mathfrak{g}=\mathfrak{s l}_{2 n+1}$ and $\sigma$ is inner.

Proof. (1) If $\mathfrak{g}_{0}$ is semisimple, then $e$ is even by Lemma 3.2.

(2) If $\mathfrak{g}_{0}$ is not semisimple, then $\sigma$ is inner and $\mathfrak{g}_{0}$ is a Levi subalgebra of a (maximal) 
parabolic subalgebra with abelian nilradical. Namely, there is $\beta \in \Pi$ such that $[\theta: \beta]=1$ and the set of simple roots of $\mathfrak{g}_{0}$ is $\Pi_{0}:=\Pi \backslash\{\beta\}$. Set $\Delta_{\beta}(i)=\{\gamma \in \Delta \mid[\gamma: \beta]=i\}$. Then $\Delta=\Delta_{\beta}(-1) \cup \Delta_{\beta}(0) \cup \Delta_{\beta}(1)$,

$$
\mathfrak{g}_{0}=\mathfrak{h} \oplus\left(\bigoplus_{\gamma \in \Delta_{\beta}(0)} \mathfrak{g}_{\gamma}\right) \text { and } \mathfrak{g}_{1}=\bigoplus_{\gamma \in \Delta_{\beta}(-1) \cup \Delta_{\beta}(1)} \mathfrak{g}_{\gamma}=\mathfrak{g}(-1) \oplus \mathfrak{g}(1)
$$

Here $\mathfrak{g}(1)$ is a simple $\mathfrak{g}_{0}$-module, with the highest (resp. lowest) weight $\theta$ (resp. $\beta$ ) w.r.t. $\Delta_{\beta}(0)^{+}=\Delta_{\beta}(0) \cap \Delta^{+}$. If $\theta=\beta+\sum_{\alpha_{i} \in \Pi_{0}} n_{i} \alpha_{i}$, then $\mathrm{c}(\mathfrak{g})=\sum n_{i}+2$. Let $\{e, h, f\}$ be a principal $\mathfrak{s l}_{2}$-triple in $\left[\mathfrak{g}_{0}, \mathfrak{g}_{0}\right] \subset \mathfrak{g}_{0}$ such that $h \in \mathfrak{h}$ and $e=\sum_{\alpha_{i} \in \Pi_{0}} e_{\alpha_{i}}$. Then $\alpha_{i}(h)=2$ for all $\alpha_{i} \in \Pi_{0}$ and $\theta(h)=-\beta(h)$. It follows that $\theta(h)=\sum n_{i}=\mathrm{c}(\mathfrak{g})-2$. Thus, the eigenvalue $\theta(h)$ is even if and only if $\mathbf{c}(\mathfrak{g})$ is even. In this case all $h$-eigenvalues in $\mathfrak{g}_{1}$ are even (Lemma 3.1) and hence $e$ is even in $\mathfrak{g}$. It remains to observe that $\mathrm{c}(\mathfrak{g})$ is odd if and only if $\mathfrak{g}=\mathfrak{s l}_{2 n+1}$.

Let $\kappa(\mathfrak{g})$ denote the maximal number of pairwise disjoint nodes in the Dynkin diagram of $\mathfrak{g}$. By Theorem 3.5, if $\left\{e_{\sigma}, h_{\sigma}, f_{\sigma}\right\}$ is a principal $\mathfrak{s l}_{2}$-triple in $\mathfrak{g}_{0}=\mathfrak{g}^{\sigma}$ for some $\sigma \in \operatorname{lnv}(\mathfrak{g})$, then $\operatorname{dim} \mathfrak{g}^{h_{\sigma}} \leqslant \mathrm{rk}(\mathfrak{g})+2 \kappa(\mathfrak{g})$. We prove in Section 4 that, for $\mathfrak{g} \neq \mathfrak{s l}_{2 n+1}$, there is always an inner involution $\vartheta$ such that $\operatorname{dim} \mathfrak{g}^{h_{\vartheta}}=\mathrm{rk}(\mathfrak{g})+2 \kappa(\mathfrak{g})$ and hence $\mathcal{D}\left(e_{\vartheta}\right)$ has the maximal possible number of isolated zeros.

Remark 3.7. It is readily seen that if $\mathfrak{g} \neq \mathbf{D}_{2 n}$, then $\kappa(\mathfrak{g})=\left[\frac{\mathrm{rk}(\mathfrak{g})+1}{2}\right]$, while $\kappa\left(\mathbf{D}_{2 n}\right)=n+1$. A uniform but more fancy expression that can also be verified case-by-case is

$$
\kappa(\mathfrak{g})=\#\left\{\gamma \in \Delta^{+} \mid \operatorname{ht}(\gamma)=[(\mathrm{c}(\mathfrak{g})+1) / 2]=: a\right\} .
$$

Note that $\bigoplus_{\gamma: \text { ht }(\gamma) \geqslant a} \mathfrak{g}_{\gamma}$ is an abelian ideal of the Borel subalgebra $\mathfrak{b}=\mathfrak{h} \oplus\left(\bigoplus_{\gamma \in \Delta^{+}} \mathfrak{g}_{\gamma}\right)$. Using a result of Sommers related to the theory of ad-nilpotent ideals of $\mathfrak{b}$ [18, Theorem 6.4], one obtains inequality " $\geqslant$ " in (3.3). Moreover, if $c(\mathfrak{g})$ is even, which only excludes $\mathfrak{g}=\mathfrak{s l}_{2 n+1}$, then I can give a case-free proof of $(3 \cdot 3)$.

Assume that $e \in \mathfrak{g}_{0}=\mathfrak{g}^{\sigma}$ is even in $\mathfrak{g}$, and let $\mathfrak{g}=\bigoplus_{i, j} \mathfrak{g}_{j}(2 i)$ be a $(\sigma, e)$-grading. Recall that $d_{j}(i)=\operatorname{dim} \mathfrak{g}_{j}(i), d_{0}(0) \geqslant d_{j}(i)$ for $i \neq 0$ (Lemma 3.4), and $d_{j}(i) \geqslant d_{j}(i+2)$ for $i \geqslant 0$, cf. Eq. (3.2). Consider the even integers $m_{j}=\max \left\{k \mid d_{j}(k) \neq 0\right\}$ for $j=0,1$.

Proposition 3.8. Given $\sigma \in \operatorname{lnv}(\mathfrak{g})$ and $a(\sigma, e)$-grading of $\mathfrak{g}$, suppose that $d_{0}(0)=d_{1}(2)$. Then

(1) $G(0) \cdot e \cap \mathfrak{g}_{1}(2) \neq \varnothing$. In particular, $G \cdot e \cap \mathfrak{g}_{1} \neq \varnothing$;

(2) $e$ is almost distinguished in $\mathfrak{g}$;

(3) $\left|m_{0}-m_{1}\right| \leqslant 2$ and $d_{0}(0) \leqslant d_{1}(0)$.

Proof. (1) For $e^{\prime} \in \mathfrak{g}_{1}(2)$, the space $\left[\mathfrak{g}_{1}(-2), e^{\prime}\right]$ is the orthogonal complement of $\mathfrak{g}_{0}^{e^{\prime}}(0)$ in $\mathfrak{g}_{0}(0)$ w.r.t. the Killing form. Let $\mathcal{O}$ be the dense $G_{0}(0)$-orbit in $\mathfrak{g}_{1}(2)$. If $e^{\prime} \in \mathcal{O}$, then $\mathfrak{g}_{0}^{e^{\prime}}(0)=\{0\}$ for the dimension reason. Hence $\left[\mathfrak{g}_{1}(-2), e^{\prime}\right]=\mathfrak{g}_{0}(0) \ni h$. That is, there is 
$f^{\prime} \in \mathfrak{g}_{1}(-2)$ such that $\left\{e^{\prime}, h, f^{\prime}\right\}$ is an $\mathfrak{s l}_{2}$-triple. Because $G(0)$ is the centraliser of $h$ in $G$, this also implies that $e^{\prime} \in G(0) \cdot e$, see [23, Theorem 1(4)].

(2) Since both ad $e^{\prime}: \mathfrak{g}_{0}(0) \rightarrow \mathfrak{g}_{1}(2)$ and ad $e^{\prime}: \mathfrak{g}_{1}(0) \rightarrow \mathfrak{g}_{0}(2)$ are onto and $d_{0}(0)=d_{1}(2)$, we see that $\mathfrak{g}(0)^{e^{\prime}}=\mathfrak{g}_{\text {red }}^{e^{\prime}} \in \mathfrak{g}_{1}(0)$. Hence $\mathfrak{g}_{\text {red }}^{e^{\prime}}$ is a toral subalgebra, and so is $\mathfrak{g}_{\text {red }}^{e}$.

(3) Using the $\mathfrak{s l}_{2}$-triple $\left\{e^{\prime}, h, f^{\prime}\right\}$ with $e^{\prime} \in \mathfrak{g}_{1}(2)$, we see that ad $e^{\prime}$ takes $\mathfrak{g}_{j}(i)$ to $\mathfrak{g}_{j+1}(i+2)$ and ad $e^{\prime}: \mathfrak{g}(\geqslant 0) \rightarrow \mathfrak{g}(\geqslant 2)$ is onto. Since $\mathfrak{g}_{0}\left(m_{0}\right)$ and $\mathfrak{g}_{1}\left(m_{1}\right)$ are in the range of ad $e^{\prime}$, one has $\mathfrak{g}_{0}\left(m_{1}-2\right) \neq 0$ and $\mathfrak{g}_{1}\left(m_{0}-2\right) \neq 0$, i.e., $\left|m_{0}-m_{1}\right| \leqslant 2$. Note also that $d_{1}(0) \geqslant d_{1}(2)=$ $d_{0}(0)$.

The hypothesis of Proposition 3.8 is rather restrictive. It means that the total number of $\langle e, h, f\rangle$-modules in $\mathfrak{g}_{0}$ equals the number of nontrivial $\langle e, h, f\rangle$-modules in $\mathfrak{g}_{1}$, i.e., roughly speaking, $\mathfrak{g}_{0}$ cannot be much bigger than $\mathfrak{g}_{1}$. Actually, assertions of Proposition 3.8 fail, if $\mathfrak{g}_{0}$ is considerably larger than $\mathfrak{g}_{1}$. For instance, let $e \in \mathfrak{g}_{0}$ be regular in $\mathfrak{g}_{0}$ for $\left(\mathfrak{g}, \mathfrak{g}_{0}\right)=\left(\mathfrak{s o}_{2 n}, \mathfrak{s o}_{2 n-1}\right)$ with $n \geqslant 3$. Then $d_{0}(0)=n-1, d_{1}(0)=d_{1}(2)=1, m_{0}=4 n-6$, and $m_{1}=2 n-2$. In this case, we also have $G \cdot e \cap \mathfrak{g}_{1}=\varnothing$. The same conclusions hold for $\left(\mathfrak{s o}_{2 n+1}, \mathfrak{s o}_{2 n}\right)$ as well.

For $e \in \mathfrak{g}_{0}$, it can happen that $G \cdot e \cap \mathfrak{g}_{1} \neq \varnothing$, while $G(0) \cdot e \cap \mathfrak{g}_{1}(2)=\varnothing$. By the construction, we have $e \in \mathfrak{g}_{0}(2) \subset \mathfrak{g}(2)$ and $G(0) \cdot e$ is the dense orbit in $\mathfrak{g}(2)$. But this dense $G(0)$-orbit does not necessarily meet $\mathfrak{g}_{1}(2)$. A simple possible reason for that is that $\mathfrak{g}_{0}(2)$ contains a 1-dimensional $G(0)$-module.

Proposition 3.9. Given $\sigma \in \operatorname{Inv}(\mathfrak{g})$ and $e \in \mathfrak{g}_{0, \text { reg }} \cap \mathcal{N}$, suppose that $G \cdot e \cap \mathfrak{g}_{1}^{\left(\sigma^{\prime}\right)} \neq \varnothing$ for some $\sigma^{\prime} \in \operatorname{Inv}(\mathfrak{g})$. Then the Satake diagram $\operatorname{Sat}\left(\sigma^{\prime}\right)$ has only isolated black nodes (IBN for short). Moreover, the set of black nodes of $\operatorname{Sat}\left(\sigma^{\prime}\right)$ is contained in the set zeros of $\mathcal{D}(e)$.

Proof. Set $\tilde{\mathfrak{g}}_{i}=\mathfrak{g}_{i}^{\left(\sigma^{\prime}\right)}$. If $x \in \tilde{\mathfrak{g}}_{1}$ is a generic semisimple element, then Sat $\left(\sigma^{\prime}\right)$ has IBN if and only if $\left[\mathfrak{g}^{x}, \mathfrak{g}^{x}\right] \simeq\left(\mathfrak{s l}_{2}\right)^{k^{\prime}}$ for some $k^{\prime}$ (and then Sat $\left(\sigma^{\prime}\right)$ has exactly $k^{\prime}$ isolated black nodes).

Take any $e^{\prime} \in G \cdot e \cap \tilde{\mathfrak{g}}_{1}$. There is an $\mathfrak{s l}_{2}$-triple $\left\{e^{\prime}, h^{\prime}, f^{\prime}\right\}$ such that $h^{\prime} \in \tilde{\mathfrak{g}}_{0}$ and $f^{\prime} \in \tilde{\mathfrak{g}}_{1}$ [10]. Here $\tilde{h}=e^{\prime}+f^{\prime}$ is $S L_{2}$-conjugate to $h^{\prime}$ in $\left\langle e^{\prime}, h^{\prime}, f^{\prime}\right\rangle \simeq \mathfrak{s l}_{2}$ and hence in $\mathfrak{g}$. Since $e^{\prime} \in G \cdot e$, one also has $h^{\prime} \in G \cdot h$. Therefore $\tilde{h} \in G \cdot h$. Since $\left[\mathfrak{g}^{h}, \mathfrak{g}^{h}\right] \simeq\left(\mathfrak{s l}_{2}\right)^{k}$ for some $k$ (Theorem 3.5), we have thus detected a semisimple element $\tilde{h} \in \tilde{\mathfrak{g}}_{1}$ such that

$$
\left[\tilde{\mathfrak{g}}_{0}^{\tilde{h}}, \tilde{\mathfrak{g}}_{0}^{\tilde{h}}\right] \subset\left[\mathfrak{g}^{\tilde{h}}, \mathfrak{g}^{\tilde{h}}\right] \simeq\left(\mathfrak{s l}_{2}\right)^{k} .
$$

Since $\tilde{h}$ is semisimple, the $\tilde{G}_{0}$-orbit of $\tilde{h}$ is closed in $\tilde{\mathfrak{g}}_{1}$. For a generic semisimple $x \in \tilde{\mathfrak{g}}_{1}$, it then follows from Luna's slice theorem [12, III.3] that the stabiliser $\tilde{\mathfrak{g}}_{0}^{x}$ is $\tilde{G}_{0}$-conjugate to a subalgebra of $\tilde{\mathfrak{g}}_{0}^{\tilde{h}}$. Therefore, $\left[\mathfrak{g}^{x}, \mathfrak{g}^{x}\right]=\left[\tilde{\mathfrak{g}}_{0}^{x}, \tilde{\mathfrak{g}}_{0}^{x}\right] \simeq\left(\mathfrak{s l}_{2}\right)^{k^{\prime}}$ for some $k^{\prime} \leqslant k$.

Combining Propositions 3.8(1) and 3.9, we obtain 
Corollary 3.10. Suppose that $e \in \mathfrak{g}_{0, \text { reg }} \cap \mathcal{N}$ and the $(\sigma, e)$-grading satisfies the condition that $d_{0}(0)=d_{1}(2)$. Then Sat $(\sigma)$ has only IBN and the black nodes of Sat $(\sigma)$ are contained among the zeros of $\mathcal{D}(e)$.

The complete list of $\sigma \in \operatorname{Inv}(\mathfrak{g})$ such that $\mathfrak{g}$ is simple and $d_{0}(0)=d_{1}(2)$ for $e \in \mathfrak{g}_{0, \text { reg }} \cap \mathcal{N}$ is as follows. We point out the pairs $\left(\mathfrak{g}, \mathfrak{g}_{0}\right)$.

1) $\sigma=\vartheta_{\text {max }}$ for $\mathfrak{g} \neq \mathfrak{s p}_{4 n+2}$, i.e., $\left(\mathfrak{s l}_{n}, \mathfrak{s o}_{n}\right),\left(\mathfrak{s o}_{2 k}, \mathfrak{s o}_{k} \dot{+} \mathfrak{s o}_{k}\right),\left(\mathfrak{s o}_{2 k+1}, \mathfrak{s o}_{k+1} \dot{+} \mathfrak{s o}_{k}\right),\left(\mathfrak{s p}_{4 n}, \mathfrak{g l}_{2 n}\right)$, $\left(\mathbf{E}_{6}, \mathbf{C}_{4}\right),\left(\mathbf{E}_{7}, \mathbf{A}_{7}\right),\left(\mathbf{E}_{8}, \mathbf{D}_{8}\right),\left(\mathbf{F}_{4}, \mathbf{C}_{3} \dot{+} \mathbf{A}_{1}\right),\left(\mathbf{G}_{2}, \mathbf{A}_{1} \dot{+} \mathbf{A}_{1}\right)$.

2) the others: $\left(\mathfrak{s o}_{2 k}, \mathfrak{s o}_{k+1} \dot{+} \mathfrak{s o}_{k-1}\right),\left(\mathfrak{s o}_{4 k+1}, \mathfrak{s o}_{2 k+2} \dot{+} \mathfrak{s o}_{2 k-1}\right),\left(\mathbf{E}_{6}, \mathbf{A}_{5} \dot{+} \mathbf{A}_{1}\right)$.

\section{A PRINCIPAL INNER INVOLUTION AND THE $B$-ACTION ON $[\mathfrak{u}, \mathfrak{u}]$}

For a fixed choice of $\mathfrak{h} \subset \mathfrak{g}$ and $\Delta^{+} \subset \Delta=\Delta(\mathfrak{g}, \mathfrak{h})$, the principal $\mathbb{Z}$-grading $\mathfrak{g}=\bigoplus_{i \in \mathbb{Z}} \mathfrak{g}\langle i\rangle$ is defined by the conditions that $\mathfrak{g}\langle 0\rangle=\mathfrak{h}$ and $\mathfrak{g}\langle i\rangle=\bigoplus_{\gamma: \mathfrak{h t}(\gamma)=i} \mathfrak{g}_{\gamma}$ for $i \neq 0$. Then $\mathfrak{b}=\mathfrak{g}\langle\geqslant 0\rangle$ is a Borel subalgebra, $\mathfrak{u}=[\mathfrak{b}, \mathfrak{b}]=\mathfrak{g}\langle\geqslant 1\rangle$, and $\mathfrak{u}^{\prime}=\mathfrak{g}\langle\geqslant 2\rangle$. Accordingly, we set $\Delta\langle i\rangle:=$ $\{\gamma \in \Delta \mid \mathrm{ht}(\gamma)=i\}$ for $i \neq 0$. In particular, $\Delta\langle 1\rangle=\Pi$ is the set of simple roots in $\Delta^{+}$. (Alternatively, one can say that this $\mathbb{Z}$-grading is determined by a principal $\mathfrak{s i}_{2}$-triple.)

For $\gamma \in \Delta$, let $e_{\gamma} \in \mathfrak{g}_{\gamma}$ be a nonzero root vector. By a classical result of Kostant [9, Theorem 5.3], a nilpotent element $v=\sum_{\gamma \in \Pi} c_{\gamma} e_{\gamma} \in \mathfrak{g}\langle 1\rangle$ is regular in $\mathfrak{g}$ if and only if $c_{\gamma} \neq 0$ for all $\gamma \in \Pi$. In this case, the $B$-orbit $B \cdot v$ is dense in $\mathfrak{u}$. Define the subspaces

$$
\mathfrak{g}_{\langle 0\rangle}:=\bigoplus_{i \text { even }} \mathfrak{g}\langle i\rangle, \quad \mathfrak{g}_{\langle 1\rangle}:=\bigoplus_{i \text { odd }} \mathfrak{g}\langle i\rangle
$$

This provides the $\mathbb{Z}_{2}$-grading $\mathfrak{g}=\mathfrak{g}_{\langle 0\rangle} \oplus \mathfrak{g}_{\langle 1\rangle}$, and the corresponding involution of $\mathfrak{g}$ is denoted by $\vartheta$. The $G$-conjugacy class of $\vartheta$ is uniquely determined by two properties (cf. [13, Theorem 2.3]:

1) $\vartheta$ is inner (because $\left.\mathrm{rk}\left(\mathfrak{g}_{\langle 0\rangle}\right)=\mathrm{rk}(\mathfrak{g})\right)$,

2) $\mathfrak{g}_{\langle 1\rangle}=\mathfrak{g}_{1}^{(\vartheta)}$ contains a regular nilpotent element of $\mathfrak{g}$ (because $\left.v \in \mathfrak{g}\langle 1\rangle \subset \mathfrak{g}_{\langle 1\rangle}\right)$.

We say that $\vartheta$ is a principal inner involution (=PI-involution). A PI-involution of $\mathfrak{g}$ is of maximal rank if and only if $\mathfrak{g} \neq \mathbf{A}_{n}, \mathbf{D}_{2 n+1}, \mathbf{E}_{6}$.

Here $\mathfrak{b}_{\langle 0\rangle}=\mathfrak{b} \cap \mathfrak{g}_{\langle 0\rangle}$ is a Borel subalgebra of $\mathfrak{g}_{\langle 0\rangle}$ and $\mathfrak{u} \cap \mathfrak{g}_{\langle 0\rangle}=\mathfrak{u}^{\prime} \cap \mathfrak{g}_{\langle 0\rangle}$ is the nilradical of $\mathfrak{b}_{\langle 0\rangle}$. The roots system of $\left(\mathfrak{g}_{\langle 0\rangle}, \mathfrak{h}\right)$ is $\Delta_{e v}:=\{\gamma \in \Delta \mid \mathrm{ht}(\gamma)$ is even $\}$. Clearly, $\Delta_{e v}^{+}:=\Delta_{e v} \cap \Delta^{+}$ is a set of positive roots in $\Delta_{e v}$, and $\Delta\langle 2\rangle$ is a part of the set of simple roots in $\Delta_{e v}^{+}$. In order to prove that $\mathfrak{u}^{\prime}$ contains a dense $B$-orbit, we first attempt to test $e=\sum_{\gamma \in \Delta\langle 2\rangle} e_{\gamma}$. However, this does not always work. If such an $e$ belongs to the dense $B$-orbit in $\mathfrak{u}^{\prime}$, then $[\mathfrak{b}, e]=\mathfrak{u}^{\prime}$ and hence $\left[\mathfrak{b}_{\langle 0\rangle}, e\right]=\mathfrak{u}^{\prime} \cap \mathfrak{g}_{\langle 0\rangle}$. Therefore, e must be a regular nilpotent element of $\mathfrak{g}_{\langle 0\rangle}$ and $\Delta\langle 2\rangle$ must be the whole set of simple roots in $\Delta_{e v}^{+}$. If $\mathfrak{g}$ is simple and $\mathrm{rk}(\mathfrak{g})=r$, then $\# \Delta\langle 2\rangle=r-1$. Hence $\Delta\langle 2\rangle$ is a set of simple roots of $\Delta_{e v}$ if and only if $\mathrm{rk}\left(\left[\mathfrak{g}_{\langle 0\rangle}, \mathfrak{g}_{\langle 0\rangle}\right]\right)=r-1$, i.e., the centre of $\mathfrak{g}_{\langle 0\rangle}$ is one-dimensional.

As is well known, if $\left(\mathfrak{g}, \mathfrak{g}_{0}\right)$ is a symmetric pair and $\mathfrak{g}$ is simple, then either 
(a) $\mathfrak{g}_{0}$ is semisimple (and $\mathfrak{g}_{1}$ is a simple $\mathfrak{g}_{0}$-module); or

$(\mathfrak{B}) \mathfrak{g}_{0}$ has a one-dimensional centre (and $\mathfrak{g}_{1}$ is a sum of two simple dual $\mathfrak{g}_{0}$-modules).

For the PI-involutions, both possibilities occur. Namely, $\mathfrak{g}_{\langle 0\rangle}$ is semisimple if and only if $\theta$ is fundamental if and only if $\mathfrak{g}$ is not of type $\mathbf{A}_{r}$ or $\mathbf{C}_{r}$. These two possibilities are considered separately below.

Case $(\mathfrak{A})$. Since $\mathfrak{g}_{\langle 0\rangle}$ is semisimple and $\vartheta$ is inner, we have $\operatorname{rk}\left(\Delta_{e v}\right)=r$. Hence there is a unique minimal root $\beta$, with $\mathrm{ht}(\beta)=2 k \geqslant 4$, that is not contained in the linear span of $\Delta\langle 2\rangle$. Then $\tilde{\Pi}:=\Delta\langle 2\rangle \cup\{\beta\}$ is the set of simple roots in $\Delta_{e v}^{+}$. (Actually, $\mathrm{ht}(\beta)=4$, see Remark 4.3, but we do not need this now.) Accordingly, $\tilde{e}:=e_{\beta}+\sum_{\gamma \in \Delta\langle 2\rangle} e_{\gamma}=e_{\beta}+e$ is a regular nilpotent element of $\mathfrak{g}_{\langle 0\rangle}$.

Theorem 4.1. Suppose that $\mathfrak{g}_{\langle 0\rangle}=\mathfrak{g}^{\vartheta}$ is semisimple. As above, let $\tilde{e}=e_{\beta}+e$ be a regular nilpotent element of $\mathfrak{g}_{\langle 0\rangle}$. Then

(i) $\mathfrak{u} \cap \mathfrak{g}^{\tilde{e}}=\mathfrak{g}_{\text {nil }}^{\tilde{e}}$ and $\operatorname{dim} \mathfrak{g}_{\text {nil }}^{\tilde{e}}=2 r=2 \mathrm{rk}(\mathfrak{g})$;

(ii) the orbit $B \cdot \tilde{e}$ is dense in $\mathfrak{u}^{\prime}, \mathfrak{b} \cap \mathfrak{g}^{\tilde{e}}=\mathfrak{u} \cap \mathfrak{g}^{\tilde{e}}$, and $\mathfrak{g}^{\tilde{e}} \subset \mathfrak{g}\langle\geqslant-1\rangle$.

Proof. We have $[\mathfrak{b}, \tilde{e}] \subset \mathfrak{u}^{\prime}=\mathfrak{g}\langle\geqslant 2\rangle$. Since the roots in $\tilde{\Pi}$ are linearly independent,

$$
[\mathfrak{h}, \tilde{e}]=\bigoplus_{\gamma \in \tilde{\Pi}} \mathfrak{g}_{\gamma}=\mathfrak{g}\langle 2\rangle \oplus \mathfrak{g}_{\beta} .
$$

By the very definition of $\beta$, the space $[\mathfrak{g}\langle 2\rangle, e] \subset \mathfrak{g}\langle 4\rangle$ does not contain $\mathfrak{g}_{\beta}$. Therefore, $[\mathfrak{u}, \tilde{e}] \subset \mathfrak{g}\langle\geqslant 3\rangle \ominus \mathfrak{g}_{\beta}$. (The latter is the sum of all root spaces in $\mathfrak{g}\langle\geqslant 3\rangle$ except $\mathfrak{g}_{\beta}$.) Hence,

$$
\operatorname{dim}[\mathfrak{u}, \tilde{e}] \leqslant \operatorname{dim} \mathfrak{g}\langle\geqslant 3\rangle-1=\operatorname{dim} \mathfrak{u}-2 r .
$$

On the other hand, $\operatorname{dim} \mathfrak{g}_{\text {nil }}^{\tilde{e}} \leqslant 2 r$ (Theorem 3.5) and $\mathfrak{u} \cap \mathfrak{g}^{\tilde{e}} \subset \mathfrak{g}_{\text {nil }}^{\tilde{e}}$, since $\tilde{e}$ is almost distinguished in $\mathfrak{g}$ by Lemma 3.2. Hence

$$
\operatorname{dim}[\mathfrak{u}, \tilde{e}]=\operatorname{dim} \mathfrak{u}-\operatorname{dim}\left(\mathfrak{u} \cap \mathfrak{g}^{\tilde{e}}\right) \geqslant \operatorname{dim} \mathfrak{u}-\operatorname{dim} \mathfrak{g}_{\text {nil }}^{\tilde{e}} \geqslant \operatorname{dim} \mathfrak{u}-2 r .
$$

Consequently, there are equalities everywhere in (4.2) and (4.3), which yields (i). One also has $[\mathfrak{b}, \tilde{e}]=[\mathfrak{h}, \tilde{e}] \oplus[\mathfrak{u}, \tilde{e}]=\mathfrak{g}\langle\geqslant 2\rangle=\mathfrak{u}^{\prime}$, i.e., $B \cdot \tilde{e}$ is dense in $\mathfrak{u}^{\prime}$. Hence $\operatorname{dim}\left(\mathfrak{b} \cap \mathfrak{g}^{\tilde{e}}\right)=2 r$ and $\mathfrak{b} \cap \mathfrak{g}^{\tilde{e}}=\mathfrak{u} \cap \mathfrak{g}^{\tilde{e}}$. Finally, $[\mathfrak{g}, \tilde{e}] \supset \mathfrak{g}\langle\geqslant 2\rangle$, hence $\mathfrak{g}^{\tilde{e}} \subset \mathfrak{g}\langle\geqslant 2\rangle^{\perp}=\mathfrak{g}\langle\geqslant-1\rangle$.

Corollary 4.2. The weighted Dynkin diagram $\mathcal{D}(\tilde{e})$ contains $\kappa(\mathfrak{g})$ isolated zeros.

Proof. By Lemma 3.2, e is even in $\mathfrak{g}$. Let $\tilde{h} \in \mathfrak{h}$ be a characteristic of $\tilde{e}$ and $\mathfrak{g}=\bigoplus_{i \in \mathbb{Z}} \mathfrak{g}(2 i)$ the corresponding $\mathbb{Z}$-grading of $\mathfrak{g}$. Using Theorem 3.5(1) with $h=\tilde{h}$, we obtain

$$
\operatorname{rk}(\mathfrak{g})+2 k=\operatorname{dim} \mathfrak{g}^{\tilde{h}}=\operatorname{dim} \mathfrak{g}(0) \geqslant \operatorname{dim} \mathfrak{g}(2)=\operatorname{dim} \mathfrak{g}_{\text {nil }}^{\tilde{e}}=2 \operatorname{rk}(\mathfrak{g}),
$$

where $k$ is the number of zeros in $\mathcal{D}(\tilde{e})$. Hence $k \geqslant\left[\frac{\mathrm{rk}(\mathfrak{g})+1}{2}\right]$, which proves the assertion for $\mathfrak{g} \neq \mathbf{D}_{2 n}$ (cf. Remark 3.7). If $\mathfrak{g}=\mathbf{D}_{2 n}$, then $\mathfrak{g}_{\langle 0\rangle}=\mathbf{D}_{n}+\mathbf{D}_{n}$ and $\boldsymbol{\lambda}(\tilde{e})=(2 n-1,2 n-1,1,1)$. 
The description of $\mathcal{D}(\tilde{e})$ via partitions $[3,5.3]$ shows that the number of zeros equals $n+1$,

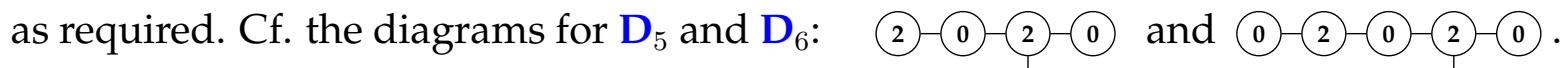

Remark 4.3. If $\mathfrak{g}_{\langle 0\rangle}=\mathfrak{g}^{\vartheta}$ is semisimple, then, beside $\Delta\langle 2\rangle$, one more root $\beta$ is needed for the basis of $\Delta_{e v}^{+}$. For $\mathbf{D}_{r}$ and $\mathbf{E}_{r}$, this $\beta$ is obtained as follows. If $\delta \in \Pi$ corresponds to the branching node in the Dynkin diagram and $\delta_{1}, \delta_{2}, \delta_{3}$ are the adjacent simple roots, then

$$
\beta=\delta+\delta_{1}+\delta_{2}+\delta_{3}
$$

see the diagram $\cdots-\overbrace{\delta_{3}}^{\delta_{1}} \overbrace{}^{\delta_{2}}-\cdots$. Obviously, $\beta$ is not a sum of two roots of height 2.

In the non-simply-laced cases, it is convenient to think that $\delta$ is the unique long simple root that has an adjacent short simple root. Using the numbering of $\Pi$ adopted in [24], $\delta$ equals $\alpha_{r-1}$ for $\mathbf{B}_{r} ; \alpha_{3}$ for $\mathbf{F}_{4}, \alpha_{2}$ for $\mathbf{G}_{2}$. Then one similarly has $\beta=\delta+\sum \frac{(\delta, \delta)}{(\alpha, \alpha)} \alpha$, where the sum ranges over all $\alpha \in \Pi$ adjacent to $\delta$. That is,

$$
\beta=\left\{\begin{array}{lll}
\alpha_{r-2}+\alpha_{r-1}+2 \alpha_{r} & \text { for } \mathbf{B}_{r} & \text { ( } \left.\alpha_{r} \text { is short }\right), \\
2 \alpha_{2}+\alpha_{3}+\alpha_{4} & \text { for } \mathbf{F}_{4} & \text { ( } \left.\alpha_{2} \text { is short }\right), \\
3 \alpha_{1}+\alpha_{2} & \text { for } \mathbf{G}_{2} & \left(\alpha_{1} \text { is short }\right) .
\end{array}\right.
$$

Thus, $\beta$ is long and $\mathrm{ht}(\beta)$ equals 4 . Under the usual unfolding procedure $\mathbf{B}_{r} \leadsto \mathbf{D}_{r+1}$, $\mathbf{F}_{4} \leadsto \mathbf{E}_{6}$, and $\mathbf{G}_{2} \leadsto \mathbf{D}_{4}$, this $\delta$ gives rise to the simple root associated with the branching node and $\beta$ of (4.5) transforms into $\beta$ of (4.4).

Since $\tilde{e}$ is regular in $\mathfrak{g}_{\langle 0\rangle}$, one has $\gamma(\tilde{h})=2$ for all $\gamma \in \Delta\langle 2\rangle \cup\{\beta\}$. This allows us to determine $\mathcal{D}(\tilde{e})$ (for the $G$-orbit of $\tilde{e}$ ). Namely, the above formulae for $\delta \in \Pi$ and $\beta$ provide the following uniform answer: $\delta(\tilde{h})=2$ and then one put interlacing values 0 and 2 on all remaining nodes, see Tables 1 and 2 . Clearly, this procedure provides the maximal possible number of isolated zeros in the weighted Dynkin diagram.

Case $(\mathfrak{B})$. Here $\operatorname{rk}\left(\Delta_{e v}\right)=r-1, \Delta\langle 2\rangle$ is the set of simple roots in $\Delta_{e v}^{+}$, and $e:=$

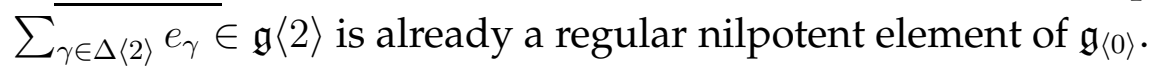

Theorem 4.4. Suppose that $\mathfrak{g}_{\langle 0\rangle}=\mathfrak{g}^{\vartheta}$ has a one-dimensional centre and $r=\mathrm{rk}(\mathfrak{g})$. Then

(i) B.e is dense in $\mathfrak{u}^{\prime}$, (ii) $\operatorname{dim}\left(\mathfrak{g}^{e} \cap \mathfrak{u}\right)=2 r-1$, (iii) $2 r-2 \leqslant \operatorname{dim} \mathfrak{g}_{\text {nil }}^{e} \leqslant 2 r-1$.

Furthermore, if e is almost distinguished in $\mathfrak{g}$, then $\operatorname{dim} \mathfrak{g}_{\text {nil }}^{e}=2 r-1$.

Proof. Such a situation occurs only if $\mathfrak{g}$ is of type $\mathbf{A}_{r}$ or $\mathbf{C}_{r}$, and it is not hard to check the assertion via direct matrix calculations. However, there is a conceptual argument, too.

(i) We have to prove that $[\mathfrak{b}, e]=\mathfrak{u}^{\prime}$, i.e., $[\mathfrak{g}\langle j-2\rangle, e]=\mathfrak{g}\langle j\rangle$ for all $j \geqslant 2$. For $j$ even, this already stems from the fact that $e \in \mathfrak{g}_{\langle 0\rangle}$ is regular nilpotent. Anyway, our next argument applies to all $j$. 
Since $e \in \mathfrak{g}\langle 2\rangle$, one can take an $\mathfrak{s l}_{2}$-triple $\{e, h, f\} \subset \mathfrak{g}_{\langle 0\rangle}$ such that $h \in \mathfrak{g}\langle 0\rangle$ and $f \in \mathfrak{g}\langle-2\rangle$, see [23]. Set $\mathfrak{g}(k)=\{v \in \mathfrak{g} \mid[h, v]=k v\}$ so that $e \in \mathfrak{g}(2)$. However, the grading $\{\mathfrak{g}(k)\}_{k \in \mathbb{Z}}$ is not always the principal $\mathbb{Z}$-grading, i.e., the spaces $\mathfrak{g}(i)$ and $\mathfrak{g}\langle i\rangle$ are different. (Actually, $\mathfrak{g}\langle k\rangle=\mathfrak{g}(k)$ for all $k$ if and only if $\mathfrak{g}$ is of type $\mathbf{A}_{2 n}$.) Still, there is a mild relationship, which is sufficient for us. Set

$$
\mathfrak{g}\langle j, k):=\mathfrak{g}\langle j\rangle \cap \mathfrak{g}(k) .
$$

Since $\mathcal{D}(e)$ has only isolated zeros by Theorem 3.5(1), if $\gamma \in \Delta^{+}$and $\operatorname{ht}(\gamma) \geqslant 2$, then $\gamma(h) \geqslant 1$. In other words, if $j \geqslant 2$ and $\mathfrak{g}\langle j, k) \neq 0$, then $k \geqslant 1$. Hence $\mathfrak{g}\langle j\rangle=\bigoplus_{k \geqslant 1} \mathfrak{g}\langle j, k)$. Since $\mathfrak{g}(k) \subset \operatorname{Im}(\operatorname{ad} e)$ for all $k \geqslant 1$ (see (i) in page 3), we see that $\mathfrak{g}\langle j\rangle$ belongs to $\operatorname{Im}(\operatorname{ad} e)$ for any $j \geqslant 2$. Hence $[\mathfrak{b}, e]=\mathfrak{u}^{\prime}$.

(ii) By part (i), we have $[\mathfrak{u}, e]=\mathfrak{g}\langle\geqslant 3\rangle$. Hence $\operatorname{dim}\left(\mathfrak{g}^{e} \cap \mathfrak{u}\right)=\operatorname{dim}(\mathfrak{g}\langle 1\rangle \oplus \mathfrak{g}\langle 2\rangle)=2 r-1$.

(iii) Combining the $\mathbb{Z}_{2}$-grading (4.1) and the $\mathbb{Z}$-grading $\{\mathfrak{g}(k)\}_{k \in \mathbb{Z}}$ determined by $h$, one obtains a mixed grading related to $(\vartheta, e)$. Here $\mathfrak{g}_{\langle 0\rangle}(1)=0$ and $\mathfrak{g}_{\langle 0\rangle}(2)=\mathfrak{g}\langle 2\rangle$; hence $\operatorname{dim} \mathfrak{g}_{\langle 0\rangle}(2)=r-1$, Next, either $\mathfrak{g}_{\langle 1\rangle}(2) \neq 0$ or $\mathfrak{g}_{\langle 1\rangle}(1) \neq 0$ (Lemma 3.1); with either dimension at most $r=\operatorname{dim} \mathfrak{g}_{\langle 0\rangle}(0)$. Hence $\operatorname{dim} \mathfrak{g}_{\text {nil }}^{e}=\operatorname{dim} \mathfrak{g}(1)+\operatorname{dim} \mathfrak{g}(2) \leqslant 2 r-1$.

On the other hand, $\operatorname{dim} \mathfrak{g}_{\langle 0\rangle}^{e}(0)=1$, i.e., $\mathfrak{g}_{\text {red }}^{e} \cap \mathfrak{g}_{\langle 0\rangle}$ is one-dimensional. This is only possible if $\left[\mathfrak{g}_{\text {red }}^{e}, \mathfrak{g}_{\text {red }}^{e}\right]$ is either trivial or $\mathfrak{s l}_{2}$. Consequently, $\operatorname{dim}\left(\mathfrak{u} \cap \mathfrak{g}^{e}\right) \leqslant \operatorname{dim} \mathfrak{g}_{\text {nil }}^{e}+1$, hence $\operatorname{dim} \mathfrak{g}_{\text {nil }}^{e} \geqslant 2 r-2$. Finally, if $e$ is almost distinguished, then $\mathfrak{u} \cap \mathfrak{g}^{e} \subset \mathfrak{g}_{\text {nil }}^{e}$ and both spaces actually coincide for dimension reason.

Remark 4.5. (1) A posteriori, there are more precise assertions related to Theorem 4.4:

- If $\mathfrak{g}=\mathbf{A}_{2 n-1}$ or $\mathbf{C}_{2 n-1}$, then $\mathfrak{g}_{\text {red }}^{e}=\mathfrak{s l}_{2}$ and $\operatorname{dim} \mathfrak{g}_{\text {nil }}^{e}=2 r-2$ (here $\left.r=2 n-1\right)$.

- If $\mathfrak{g}=\mathbf{A}_{2 n}$ or $\mathbf{C}_{2 n}$, then $\mathfrak{g}_{\text {red }}^{e}=\mathfrak{t}_{1}$ (i.e., $e$ is almost distinguished in $\mathfrak{g}$ ) and $\operatorname{dim} \mathfrak{g}_{\text {nil }}^{e}=$ $2 r-1$ (here $r=2 n$ ).

(2) The only case in which $e$ is not even in $\mathfrak{g}$ is that of $\mathbf{A}_{2 n}$ (cf. Example 3.3 and Proposition 3.6). In the remaining cases, $\mathcal{D}(e)$ has the maximal possible number of isolated zeros, see Table 2.

In Tables 1 and 2, we gather information on the PI-involutions $\vartheta$ and the nilpotent orbits of $G$ that contain a regular nilpotent element $e_{\vartheta}$ of $\mathfrak{g}_{\langle 0\rangle}$. For the exceptional (resp. classical) Lie algebras, the orbit $G \cdot e_{\vartheta}$ is denoted by the Dynkin-Bala-Carter label $[3,8.4]$ (resp. by the corresponding partition). For the exceptional Lie algebras, the structure of $\mathfrak{g}_{\text {red }}^{e_{\vartheta}}$ and the numbers $\operatorname{dim} \mathfrak{g}_{\text {nil }}^{e_{\vartheta}}$ are pointed out in the Tables in [5]. For the classical Lie algebras, this information is being extracted from the partition of $G \cdot e_{\vartheta}$, see [3, Theorem 6.1.3].

Remark 4.6. Since there is a dense $B$-orbit in $\mathfrak{u}^{\prime}$, the varieties $G \times_{B} \mathfrak{u}^{\prime}$ and $G \cdot \mathfrak{u}^{\prime}$ contain dense $G$-orbits. But their dimensions can be different, i.e., the natural 'collapsing' in the sense of Kempf [8] $\tau: G \times \times_{B} \mathfrak{u}^{\prime} \rightarrow G \cdot \mathfrak{u}^{\prime}$ is not always generically finite-to-one. Indeed, if $B \cdot e$ is dense in $\mathfrak{u}^{\prime}$, then $\operatorname{dim} G \cdot \mathfrak{u}^{\prime}=\operatorname{dim} G \cdot e$ is even and $\operatorname{dim}\left(G \times{ }_{B} \mathfrak{u}^{\prime}\right)=2 \operatorname{dim} \mathfrak{u}-r=\operatorname{dim} \mathfrak{g}-2 r$. 
TABLE 1 . The $P I$-involutions $\vartheta$ and orbits $G \cdot e_{\vartheta}$ for the exceptional Lie algebras

\begin{tabular}{|c|c|c|c|c|c|c|}
\hline $\mathfrak{g}$ & $\mathfrak{g}_{\langle 0\rangle}$ & $G \cdot e_{\vartheta}$ & $\mathcal{D}\left(e_{\vartheta}\right)$ & $\operatorname{dim} \mathfrak{g}^{e_{\vartheta}}$ & $\mathfrak{g}_{\text {red }}^{e_{\vartheta}}$ & $\operatorname{dim} \mathfrak{g}_{\text {nil }}^{e_{\vartheta}}$ \\
\hline $\mathbf{E}_{6}$ & $\mathbf{A}_{5} \dot{+} \mathbf{A}_{1}$ & $\mathrm{E}_{6}\left(a_{3}\right)$ & (2)-(0)-(2)-(2) & 12 & $\{0\}$ & 12 \\
\hline $\mathrm{E}_{7}$ & $\mathbf{A}_{7}$ & $\mathrm{E}_{6}\left(a_{1}\right)$ & (0)-2)-(1)-(2)-2) & 15 & $\mathfrak{t}_{1}$ & 14 \\
\hline $\mathrm{E}_{8}$ & $\mathbf{D}_{8}$ & $\mathrm{E}_{8}\left(a_{4}\right)$ & (2)-(0)-(2)-(0)-2-(0) & 16 & $\{0\}$ & 16 \\
\hline $\mathbf{F}_{4}$ & $\mathbf{C}_{3} \dot{+} \mathbf{A}_{1}$ & $\mathrm{~F}_{4}\left(a_{2}\right)$ & (2)-0 $=2-0$ & 8 & $\{0\}$ & 8 \\
\hline $\mathrm{G}_{2}$ & $\mathbf{A}_{1}+\widetilde{\mathbf{A}}_{1}$ & $\mathrm{G}_{2}\left(a_{1}\right)$ & $(0) \equiv 2$ & 4 & $\{0\}$ & 4 \\
\hline
\end{tabular}

TABLE 2. The PI-involutions $\vartheta$ and orbits $G \cdot e_{\vartheta}$ for the classical Lie algebras

\begin{tabular}{|c|c|c|c|c|c|c|}
\hline $\mathfrak{g}$ & $\mathfrak{g}_{\langle 0\rangle}$ & $\lambda\left(G \cdot e_{\vartheta}\right)$ & $\mathcal{D}\left(e_{\vartheta}\right)$ & $\operatorname{dim} \mathfrak{g}^{e_{\vartheta}}$ & $\mathfrak{g}_{\text {red }}^{e_{\vartheta}}$ & $\operatorname{dim} \mathfrak{g}_{\text {nil }}^{e_{\vartheta}}$ \\
\hline $\mathbf{B}_{2 n}$ & $\mathbf{B}_{n} \dot{+} \mathbf{D}_{n}$ & $(2 n+1,2 n-1,1)$ & (2)-(0) $\cdots-(2)=0$ & $4 n$ & $\{0\}$ & $4 n$ \\
\hline $\mathbf{B}_{2 n-1}$ & $\mathbf{B}_{n-1} \dot{+} \mathbf{D}_{n}$ & $(2 n-1,2 n-1,1)$ & 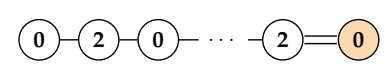 & $4 n-1$ & $\mathfrak{t}_{1}$ & $4 n-2$ \\
\hline $\mathbf{D}_{2 n}$ & $\mathbf{D}_{n} \dot{+} \mathbf{D}_{n}$ & $(2 n-1,2 n-1,1,1)$ & & $4 n+2$ & $\mathfrak{t}_{2}$ & $4 n$ \\
\hline $\mathbf{D}_{2 n-1}$ & $\mathbf{D}_{n-1} \dot{+} \mathbf{D}_{n}$ & $(2 n-1,2 n-3,1,1)$ & (2)-(0)-2 $2-\cdots-(1)-2)_{0}^{2}$ & $4 n-1$ & $\mathfrak{t}_{1}$ & $4 n-2$ \\
\hline $\mathbf{C}_{2 n-1}$ & $\mathfrak{g l}_{2 n-1}$ & $(2 n-1,2 n-1)$ & (0)-2- $-\cdots-(0)=0$ & $4 n-1$ & $\mathfrak{s l}_{2}$ & $4 n-4$ \\
\hline $\mathrm{C}_{2 n}$ & $\mathfrak{g l}_{2 n}$ & $(2 n, 2 n)$ & 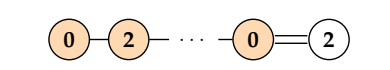 & $4 n$ & $\mathfrak{t}_{1}$ & $4 n-1$ \\
\hline $\mathbf{A}_{2 n-1}$ & $\mathfrak{g l}_{n} \dot{+} \mathfrak{s l}_{n}$ & $(n, n)$ & 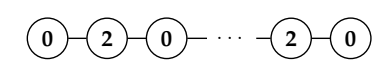 & $4 n-1$ & $\mathfrak{s l}_{2}$ & $4 n-4$ \\
\hline $\mathbf{A}_{2 n}$ & $\mathfrak{g l}_{n+1} \dot{+} \mathfrak{s l}_{n}$ & $(n+1, n)$ & (1)-(1)-1)- (1) (1) & $4 n$ & $\mathfrak{t}_{1}$ & $4 n-1$ \\
\hline
\end{tabular}

Hence the necessary (but not sufficient) condition is that $r$ is even. It is also clear that $d:=\operatorname{dim}\left(G \times \times_{B} \mathfrak{u}^{\prime}\right)-\operatorname{dim} G \cdot \mathfrak{u}^{\prime}=\operatorname{dim} \mathfrak{g}^{e_{\vartheta}}-2 r$. Hence using Tables 1 and 2, we obtain $\tau$ is generically finite-to-one if and only if $\mathrm{rk}(\mathfrak{g})$ is even and $\mathfrak{g} \neq \mathbf{D}_{2 n}$.

If $\mathrm{rk}(\mathfrak{g})$ is odd, then $d=1$, whereas $d=2$ for $\mathbf{D}_{2 n}$. 


\section{MiXED GRADINGS AND DIVISIBLE ORBITS}

For $e \in \mathcal{N}$, let $\frac{1}{2} \mathcal{D}(e)$ be the Dynkin diagram equipped with the labels $\frac{1}{2} \alpha(h), \alpha \in \Pi$. Following [14], an element $e \in \mathcal{N}$ (orbit $G \cdot e \subset \mathcal{N}$ ) is said to be divisible, if $\frac{1}{2} \mathcal{D}(e)$ is again a weighted Dynkin diagram. Equivalently, if $h$ is a characteristic of $e$, then $h / 2$ is a characteristic of another nilpotent element. Then we write $e^{\langle 2\rangle}$ for a nilpotent element with characteristic $h / 2$. Hence $\frac{1}{2} \mathcal{D}(e)=: \mathcal{D}\left(e^{\langle 2\rangle}\right)$. The notation is suggested by the equality

$$
\operatorname{dim} \operatorname{Ker}(\operatorname{ad} e)^{2}=\operatorname{dim} \operatorname{Ker}\left(\operatorname{ad} e^{\langle 2\rangle}\right)=\operatorname{dim} \mathfrak{z}_{\mathfrak{g}}\left(e^{\langle 2\rangle}\right) .
$$

Furthermore, if $\mathfrak{g}=\mathfrak{s l}_{n}$ and $e$ is divisible, then one can take $e^{\langle 2\rangle}=e^{2}$, the usual matrix power [14, Theorem 3.1]. Clearly, a divisible element must be even. If $\{e, h, f\}$ is an $\mathfrak{s l}_{2}-$ triple and $\mathfrak{g}=\bigoplus_{i \in \mathbb{Z}} \mathfrak{g}(i)$ is the $\mathbb{Z}$-grading determined by $h$, then $e$ is divisible if and only if there is an $x \in \mathfrak{g}(4)$ such that $h \in \operatorname{Im}(\operatorname{ad} x)$. In this case, $e$ is necessarily even, there is an $\mathfrak{s l}_{2}$-triple of the form $\{x, h / 2, \tilde{f}\}$ with $\tilde{f} \in \mathfrak{g}(-4)$, and one can take $e^{\langle 2\rangle}=x$.

Mixed gradings can be used for detecting divisible orbits with good properties. Suppose that $e \in \mathcal{N}$ is even and $\sigma(e)=e$ for some $\sigma \in \operatorname{lnv}(\mathfrak{g})$. Consider a mixed grading related to $(\sigma, e)$. Our intention is to find a suitable element $e^{\langle 2\rangle} \in \mathfrak{g}(4)$. Of course, this is not always possible, and a sufficient condition is given below. Recall that $d_{j}(i)=\operatorname{dim} \mathfrak{g}_{j}(i)$ and $d_{0}(0) \geqslant d_{j}(i)$ whenever $i \neq 0$.

Theorem 5.1. As above, let $\mathfrak{g}=\bigoplus_{i, j} \mathfrak{g}_{j}(i)$ be a mixed grading related to $(\sigma, e)$, hence $e \in \mathfrak{g}_{0}(2)$. Suppose that $d_{0}(0)=d_{1}(4)$. Then

(1) the orbit $G \cdot e$ is divisible and there exists $e^{\langle 2\rangle} \in \mathfrak{g}_{1}(4) \subset \mathfrak{g}(4)$;

(2) $d_{0}(0)=d_{0}(2)=d_{1}(2)=d_{1}(4)$ and also $d_{0}(4 k+2)=d_{1}(4 k+2)$ for all $k \in \mathbb{Z}$;

(3) $\mathfrak{g}_{1}$ does not contain 3-dimensional $\langle e, h, f\rangle$-modules, $\mathfrak{g}_{0}$ is semisimple, and e is distinguished in $\mathfrak{g}_{0}$;

(4) both $e$ and $e^{\langle 2\rangle}$ are almost distinguished in $\mathfrak{g}$.

Proof. (1) If $x \in \mathfrak{g}_{1}(4)$, then $\left[\mathfrak{g}_{1}(-4), x\right]$ is the orthogonal complement of $\mathfrak{g}_{0}^{x}(0)$ in $\mathfrak{g}_{0}(0)$. By Lemma 3.4, $G_{0}(0)$ has an open orbit in $\mathfrak{g}_{1}(4)$, say $\mathcal{O}$. Now, if $x \in \mathcal{O}$, then $\mathfrak{g}_{0}^{x}(0)=\{0\}$ in view of the hypothesis $d_{0}(0)=d_{1}(4)$. Hence $\left[\mathfrak{g}_{1}(-4), x\right]=\mathfrak{g}_{0}(0)$ and there is $y \in \mathfrak{g}_{1}(-4)$ such that $[x, y]=h / 2$. Then $\{x, h / 2, y\}$ a desired $\mathfrak{s l}_{2}$-triple. Thus, every element of the open $G_{0}(0)$-orbit in $\mathfrak{g}_{1}(4)$ can be taken as $e^{\langle 2\rangle}$. This also implies that $e$ must be even in $\mathfrak{g}$.

(2) Since $h / 2$ is a characteristic of $e^{\langle 2\rangle} \in \mathfrak{g}_{1}(4)$, the analogue of Eq. (3.2) for $e^{\langle 2\rangle}$ implies that the mappings ad $e^{\langle 2\rangle}: \mathfrak{g}_{0}(-2) \rightarrow \mathfrak{g}_{1}(2)$ and ad $e^{\langle 2\rangle}: \mathfrak{g}_{1}(-2) \rightarrow \mathfrak{g}_{0}(2)$ are bijective. Hence $\operatorname{dim} \mathfrak{g}_{0}(2)=\operatorname{dim} \mathfrak{g}_{1}(2)$. Combining this with Eq. (3.2) yields

$$
d_{0}(0) \geqslant d_{0}(2)=d_{1}(2) \geqslant d_{1}(4)=d_{0}(0) .
$$


Since the $\mathbb{Z}$-grading of $\mathfrak{g}$ is determined by a characteristic of $e$ and $e \in \mathfrak{g}(2)$, the $\mathfrak{s l}_{2}$-theory readily implies that $(\operatorname{ad} e)^{m}: \mathfrak{g}(-m) \rightarrow \mathfrak{g}(m)$ is a bijection. Likewise, since $h / 2$ is a characteristic of $e^{\langle 2\rangle}$ and $e^{\langle 2\rangle} \in \mathfrak{g}_{1}(4)$, this implies that

$$
\left(\operatorname{ad} e^{\langle 2\rangle}\right)^{2 k+1}: \mathfrak{g}_{0}(-4 k-2) \rightarrow \mathfrak{g}_{1}(4 k+2) \text { and }\left(\operatorname{ad} e^{\langle 2\rangle}\right)^{2 k+1}: \mathfrak{g}_{1}(-4 k-2) \rightarrow \mathfrak{g}_{0}(4 k+2)
$$

are bijections. Therefore, $d_{0}(4 k+2)=d_{1}(4 k+2)$.

(3) Now, the equality $d_{1}(2)=d_{1}(4)$ means that $\mathfrak{g}_{1}$ contains no 3-dimensional $\langle e, h, f\rangle$ modules. While the equality $d_{0}(0)=d_{0}(2)$ implies that $\mathfrak{g}_{0}$ is semisimple and $e$ is distinguished in $\mathfrak{g}_{0}$ (cf. the proof of Theorem 3.5(2)).

(4) We know that $\mathfrak{g}_{\text {red }}^{e}=\operatorname{Ker}(\operatorname{ad} e) \cap \mathfrak{g}(0)$. Since ad $e: \mathfrak{g}_{0}(0) \stackrel{\sim}{\rightarrow} \mathfrak{g}_{0}(2)$, we see that $\mathfrak{g}_{\text {red }}^{e} \subset$ $\mathfrak{g}_{1}(0)$. Hence $\mathfrak{g}_{\text {red }}^{e}$ is toral. Likewise, $\mathfrak{g}_{\text {red }}^{e(2\rangle}=\operatorname{Ker}\left(\operatorname{ad} e^{\langle 2\rangle}\right) \cap \mathfrak{g}(0)$ and ad $e^{\langle 2\rangle}: \mathfrak{g}_{0}(0) \stackrel{\sim}{\rightarrow} \mathfrak{g}_{1}(4)$. Therefore, $\mathfrak{g}_{\text {red }}^{e^{\langle 2\rangle}} \subset \mathfrak{g}_{1}(0)$ and $\mathfrak{g}_{\text {red }}^{e^{(2)}}$ is toral.

Remark 5.2. For a $(\sigma, e)$-grading, the hypothesis $d_{0}(0)=d_{1}(4)$ implies that $d_{0}(0)=d_{1}(2)$. In Proposition 3.8, we derived from the latter that $G(0) \cdot e \cap \mathfrak{g}_{1}(2) \neq \varnothing$. For a divisible orbit $G \cdot e$, it follows from [1, Theorem 1] that the conditions $G \cdot e \cap \mathfrak{g}_{1} \neq \varnothing$ and $G \cdot e^{\langle 2\rangle} \cap \mathfrak{g}_{1} \neq \varnothing$ are equivalent (cf. also Remark 2.2). However, a subtle point is that if $G \cdot e$ is divisible and $G \cdot e \cap \mathfrak{g}_{1} \neq \varnothing$, then one may not simultaneously have that $e \in \mathfrak{g}_{0}(2)$ and $e^{\langle 2\rangle} \in \mathfrak{g}_{1}(4)$. For, starting with $e \in \mathfrak{g}_{0}(2)$, we can certainly find $e^{\langle 2\rangle} \in \mathfrak{g}(4)$, but then we need a stronger condition that $G(0) \cdot e^{\langle 2\rangle} \cap \mathfrak{g}_{1}(4) \neq \varnothing$.

Using Theorem 5.1, we classify below all the pairs $(\sigma, G \cdot e)$ such that $d_{0}(0)=d_{1}(4)$.

Theorem 5.3. For a mixed grading related to $(\sigma, e)$, the equality $d_{0}(0)=d_{1}(4)$ holds exactly in the following cases:

(a) $\mathfrak{g}=\mathbf{E}_{n}, \sigma=\vartheta_{\max }$, and e is regular in $\mathfrak{g}_{0}$; here $\mathfrak{g}_{0}=\mathbf{C}_{4}, \mathbf{A}_{7}, \mathbf{D}_{8}$ and the Dynkin-BalaCarter labels of $G \cdot e$ are $\mathrm{E}_{6}\left(a_{1}\right), \mathrm{E}_{6}\left(a_{1}\right), \mathrm{E}_{8}\left(a_{4}\right)$ for $n=6,7,8$, respectively.

(b) $\mathfrak{g}=\mathfrak{s l}_{n}, \mathfrak{g}_{0}=\mathfrak{s o}_{n}$ (hence $\sigma$ is also of maximal rank), and $\boldsymbol{\lambda}(e)=\left(2 m_{1}+1, \ldots, 2 m_{s}+1\right)$ is a partition of $n$ such that $m_{i-1}-m_{i} \geqslant 2$ for $i \geqslant 2$. In particular, one obtains a regular nilpotent element of $\mathfrak{g}_{0}=\mathfrak{s o}_{2 m_{1}+1}\left(\right.$ resp. $\left.\mathfrak{s o}_{2 m_{1}+2}\right)$ if $s=1$ (resp. if $s=2$ and $\left.m_{2}=0\right)$.

Proof. 1) The "only if" part relies on the explicit description of divisible orbits. For the classical series, such a description is given in terms of partitions [14, Theorem 3.1], see also below. For the exceptional algebras, there is the list of divisible orbits [14, Table 1]. By Theorem 5.1(4), if $d_{0}(0)=d_{1}(4)$, then $e$ and $e^{\langle 2\rangle}$ are almost distinguished. Let us begin with finding the pairs of orbits $G \cdot e$ and $G \cdot e^{\langle 2\rangle}$ such that both $e, e^{\langle 2\rangle}$ are almost distinguished.

- For the exceptional algebras, Table 1 in [14], together with the known information on the reductive part of centralisers [5], shows that there are only three such pairs of orbits. This leads to the three $\mathbf{E}_{n}$-cases, see Example 5.5. 
- For $\mathfrak{s o}_{n}$ and $\mathfrak{s p}_{2 n}$, an inspection of partitions $\boldsymbol{\lambda}(e)$ of the divisible orbits shows that there are no pairs $\left(e, e^{\langle 2\rangle}\right)$ such that both orbits are almost distinguished. More precisely,

- For $\mathfrak{g}=\mathfrak{s p}_{2 n}, e$ is divisible if and only if all parts of $\boldsymbol{\lambda}(e)$ are odd and occur pairwise. This already implies that $e$ is not almost distinguished, cf. [21, IV.2.25] or [3, Theorem 6.1.3], where a description of $\mathfrak{g}_{\text {red }}^{e}$ is given in terms of $\boldsymbol{\lambda}(e)$.

- For $\mathfrak{g}=\mathfrak{s o}_{n}, e$ is divisible if and only if all parts $\lambda_{i}$ are odd and also

$$
\begin{cases}\text { if } \lambda_{2 k-1}=4 m+3, & \text { then } \lambda_{2 k}=4 m+3 ; \\ \text { if } \lambda_{2 k-1}=4 m+1>1, & \text { then } \lambda_{2 k} \in\{4 m+1,4 m-1\} ; \\ \text { if } \lambda_{2 k-1}=1, & \text { then there is no further conditions. }\end{cases}
$$

The partition $\boldsymbol{\lambda}\left(e^{\langle 2\rangle}\right)$ is obtained by the following rule applied to each pair $\left(\lambda_{2 k-1}, \lambda_{2 k}\right)$ of consecutive parts of $\boldsymbol{\lambda}(e)$ :

$$
\left\{\begin{aligned}
(\ldots, 4 m+3,4 m+3, \ldots) & \mapsto(\ldots, 2 m+2,2 m+2,2 m+1,2 m+1, \ldots) ; \\
(\ldots, 4 m+1,4 m+1, \ldots) & \mapsto(\ldots, 2 m+1,2 m+1,2 m, 2 m, \ldots) ; \\
(\ldots, 4 m+1,4 m-1, \ldots) & \mapsto(\ldots, 2 m+1,2 m, 2 m, 2 m-1, \ldots) .
\end{aligned}\right.
$$

In all cases, $\boldsymbol{\lambda}\left(e^{\langle 2\rangle}\right)$ has at least two equal even parts, which yields a subalgebra $\mathfrak{s l}_{2}=\mathfrak{s p}_{2}$ in $\mathfrak{z}_{\mathfrak{g}}\left(e^{\langle 2\rangle}\right)$.

- Let $\mathfrak{g}=\mathfrak{s l}_{n}$ and $\boldsymbol{\lambda}(e)=\left(\lambda_{1}, \ldots, \lambda_{s}\right)$, where $\lambda_{1} \geqslant \ldots \geqslant \lambda_{s}$. Then $e$ is divisible if and only if all $\lambda_{i}$ 's are odd, say $\lambda_{i}=2 m_{i}+1$; and $e$ is almost distinguished if and only if all $\lambda_{i}{ }^{\prime} \mathrm{s}$ are different, i.e., $m_{i}>m_{i+1}$. Next, the set of parts of $\boldsymbol{\lambda}\left(e^{\langle 2\rangle}\right)$ is $\left\{m_{i}+1, m_{i} \mid i=1, \ldots, s\right\}$ [14, Theorem 3.1]. Since $e^{\langle 2\rangle}$ is almost distinguished as well, all these parts must be different, too. Hence $m_{i}>m_{i+1}+1$.

Since $\mathfrak{g}_{0}$ has to be semisimple by Theorem 5.1(3), $\sigma$ is outer, i.e., $\mathfrak{g}_{0}=\mathfrak{s p}_{n}$ (if $n$ is even) or $\mathfrak{s o}_{n}$. But a partition with different odd parts does not correspond to a nilpotent orbit in $\mathfrak{s p}_{n}\left[3\right.$, Theorem 5.1.3]. Hence $\mathfrak{g}_{0}=\mathfrak{s o}_{n}$.

2) The "if" part follows by direct calculations, cf. Example 5.5 below. Let us give some details for the case of $\mathfrak{s l}_{n}=\mathfrak{s l}(\mathbb{V})$. Let $\mathrm{R}_{i}$ denote the simple $\mathfrak{s l}_{2}$-module of dimension $i+1$. If $\boldsymbol{\lambda}(e)=\left(2 m_{1}+1, \ldots, 2 m_{s}+1\right), \mathfrak{g}_{0}=\mathfrak{s o}_{n}$, and $\mathfrak{s l}_{2} \simeq\langle e, h, f\rangle \subset \mathfrak{g}_{0}$, then $\mathbb{V}=\mathrm{R}_{2 m_{1}}+\cdots+\mathrm{R}_{2 m_{s}}$ as $\mathfrak{s l}_{2}$-module. Therefore

$$
\begin{gathered}
\mathfrak{g}_{0}=\wedge^{2}\left(\mathrm{R}_{2 m_{1}}+\cdots+\mathrm{R}_{2 m_{s}}\right)=\wedge^{2} \mathrm{R}_{2 m_{1}}+\cdots+\wedge^{2} \mathrm{R}_{2 m_{s}}+\sum_{i<j} \mathrm{R}_{2 m_{i}} \otimes \mathrm{R}_{2 m_{j}}, \\
\mathfrak{g}_{1}=\mathcal{S}^{2}\left(\mathrm{R}_{2 m_{1}}+\cdots+\mathrm{R}_{2 m_{s}}\right)-\mathrm{R}_{0}=\mathcal{S}^{2} \mathrm{R}_{2 m_{1}}+\cdots+\mathcal{S}^{2} \mathrm{R}_{2 m_{s}}+\sum_{i<j} \mathrm{R}_{2 m_{i}} \otimes \mathrm{R}_{2 m_{j}}-\mathrm{R}_{0} .
\end{gathered}
$$

Using the Clebsch-Gordan formula (see e.g. [19, 3.2.4]), we see that if $m_{i}-m_{i+1} \geqslant 2$, then (1) the total number of $\mathfrak{s l}_{2}$-modules in $\mathfrak{g}_{0}$ equals the number of nontrivial $\mathfrak{s l}_{2}$-modules in $\mathfrak{g}_{1}$, i.e., $d_{0}(0)=d_{1}(2)$, and (2) $\mathfrak{g}_{1}$ contains no $\mathrm{R}_{2}$, i.e., $d_{1}(2)=d_{1}(4)$.

We also notice that here $d_{0}(0)=\sum_{j=1}^{s}(2 j-1) m_{j}+\left(\begin{array}{l}s \\ 2\end{array}\right)$ and $d_{1}(0)-d_{0}(0)=s-1$. 
Remark 5.4. The constraints on $\boldsymbol{\lambda}$ for $\mathfrak{s l}_{n}$ exclude exactly the cases with $n=2,4$. This means that only those $n$ are allowed for which $\left(\mathfrak{s l}_{n}\right)^{\sigma}=\mathfrak{s o}_{n}$ is again simple.

Example 5.5. (1) Let us provide the numbers $d_{j}(i)=\operatorname{dim} \mathfrak{g}_{j}(i)$ with $i \geqslant 0$ for the $\mathbf{E}_{n}$-cases in Theorem 5.3 .

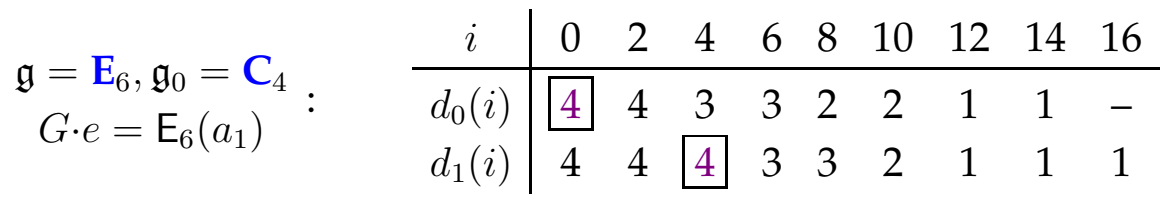

Here $\mathfrak{g}_{0} \simeq \mathrm{R}_{2}+\mathrm{R}_{6}+\mathrm{R}_{10}+\mathrm{R}_{14}$ and $\mathfrak{g}_{1} \simeq \mathrm{R}_{4}+\mathrm{R}_{8}+\mathrm{R}_{10}+\mathrm{R}_{16}$ as $\langle e, h, f\rangle$-modules.

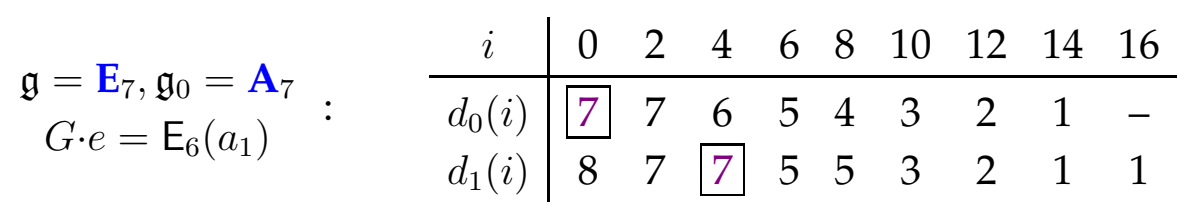

\begin{tabular}{|c|c|c|c|c|c|c|c|c|c|c|c|c|c|c|c|c|}
\hline $\mathfrak{g}=\mathbf{E}_{8}$ & $i$ & 0 & 2 & 4 & 6 & 8 & 10 & 12 & 14 & 16 & 18 & 20 & 22 & 24 & 26 & 28 \\
\hline $\mathfrak{g}_{0}=\mathbf{D}_{8}$ & $d_{0}(i)$ & 8 & 8 & 7 & 7 & 6 & 6 & 5 & 5 & 3 & 3 & 2 & 2 & 1 & 1 & \\
\hline$G \cdot e=\mathrm{E}_{8}\left(a_{4}\right)$ & $d_{1}(i)$ & 8 & 8 & 8 & 7 & 7 & 6 & 5 & 5 & 4 & 3 & 2 & 2 & 1 & 1 & \\
\hline
\end{tabular}

Here $\mathfrak{g}_{0} \simeq \sum_{i=1}^{7} \mathrm{R}_{2 i}$ and $\mathfrak{g}_{1} \simeq \mathrm{R}_{0}+2 \mathrm{R}_{4}+2 \mathrm{R}_{8}+\mathrm{R}_{10}+\mathrm{R}_{12}+\mathrm{R}_{16}$ as $\langle e, h, f\rangle$-modules.

Here $\mathfrak{g}_{0} \simeq \mathrm{R}_{2}+\mathrm{R}_{6}+\mathrm{R}_{10}+2 \mathrm{R}_{14}+\mathrm{R}_{18}+\mathrm{R}_{22}+\mathrm{R}_{26}$ and $\mathfrak{g}_{1} \simeq \mathrm{R}_{4}+\mathrm{R}_{8}+\mathrm{R}_{10}+\mathrm{R}_{14}+\mathrm{R}_{16}+\mathrm{R}_{18}+\mathrm{R}_{22}+\mathrm{R}_{28}$ as $\langle e, h, f\rangle$-modules.

(2) The following is a sample of calculations for the $\mathfrak{s l}_{n}$-case.

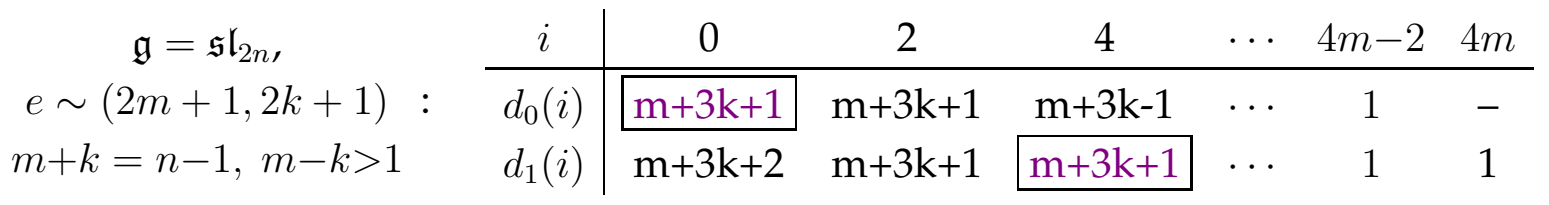

Remark 5.6. There are many examples of mixed gradings related to some $(\sigma, e)$ such that $d_{0}(0)>d_{1}(4)$ and $e \in \mathfrak{g}_{0}(2)$ is divisible in $\mathfrak{g}$. Hence there is $e^{\langle 2\rangle} \in \mathfrak{g}(4)$. However, it is then not always the case that $G \cdot e^{\langle 2\rangle} \cap \mathfrak{g}_{1}(4) \neq \varnothing$.

\section{NEW INVOLUTIONS FROM OLD ONES}

Let $\sigma$ be an involution of a simple Lie algebra $\mathfrak{g}$ and $e$ a regular nilpotent element of $\mathfrak{g}^{\sigma}=\mathfrak{g}_{0}$. If $e$ remains even in $\mathfrak{g}$, then one defines another involution having nice properties, which is denoted by $\Upsilon(\sigma)$ or $\check{\sigma}$. It is always assumed below that $e \in \mathfrak{g}_{0, \text { reg }}$ is even in $\mathfrak{g}$. By Proposition 3.6, this only excludes the inner involutions of $\mathfrak{s l}_{2 n+1}$.

Definition/construction of $\Upsilon(\sigma)=\check{\sigma}$.

Let $\mathfrak{g}=\mathfrak{g}_{0} \oplus \mathfrak{g}_{1}$ be the $\mathbb{Z}_{2}$-grading corresponding to $\sigma$ and $\{e, h, f\} \subset \mathfrak{g}_{0}$ a principal $\mathfrak{s l}_{2}$ triple in $\mathfrak{g}_{0}$. Consider the $\mathbb{Z}$-grading of $\mathfrak{g}$ determined by $h$ and, assuming that $e$ is even in 
$\mathfrak{g}$, set

$$
\mathfrak{g}_{\{0\}}=\bigoplus_{i \text { even }} \mathfrak{g}(2 i), \mathfrak{g}_{\{1\}}=\bigoplus_{i \text { odd }} \mathfrak{g}(2 i)
$$

Then $\mathfrak{g}=\mathfrak{g}_{\{0\}} \oplus \mathfrak{g}_{\{1\}}$ and $\check{\sigma}$ is the involution associated with this $\mathbb{Z}_{2}$-grading, i.e., $\mathfrak{g}_{\{i\}}=\mathfrak{g}_{i}^{(\check{\sigma})}$.

First properties of $\Upsilon$ (the passage $\sigma \mapsto \check{\sigma}$ ):

(1) $\check{\sigma}$ is inner (for, $\mathfrak{g}^{\check{\sigma}}=\mathfrak{g}_{\{0\}} \supset \mathfrak{g}_{\mathfrak{g}}(h)$, and the latter contains a Cartan subalgebra of $\mathfrak{g}$ ).

(2) $e \in \mathfrak{g}_{0}(2) \subset \mathfrak{g}_{0}$ and therefore $e \in \mathfrak{g}_{\{1\}}$.

(3) The involutions $\sigma$ and $\check{\sigma}$ commute; hence $\sigma \check{\sigma}$ is also an involution.

(4) $\sigma$ and $\sigma \check{\sigma}$ belong to the same connected component of the group $\operatorname{Aut}(\mathfrak{g})$; therefore, $\sigma$ is inner if and only if $\sigma \check{\sigma}$ is inner.

For $\mathfrak{g} \neq \mathfrak{s l}_{2 n+1}$, we think of $\Upsilon$ as a map from $\operatorname{lnv}(\mathfrak{g})$ to the set of inner involutions of $\mathfrak{g}$. Let $\mathfrak{g}=\mathfrak{g}_{[0]} \oplus \mathfrak{g}_{[1]}$ be the $\mathbb{Z}_{2}$-grading corresponding to $\sigma \check{\sigma}$, i.e., $\mathfrak{g}_{[i]}=\mathfrak{g}_{i}^{(\sigma \check{\sigma})}$. Then

$$
\mathfrak{g}^{\sigma \check{\sigma}}=\mathfrak{g}_{[0]}=\bigoplus_{i \in \mathbb{Z}} \mathfrak{g}_{\bar{i}}(2 i) \quad \text { and } \quad \mathfrak{g}_{[1]}=\bigoplus_{i \in \mathbb{Z}} \mathfrak{g}_{\overline{i+1}}(2 i)
$$

where $\bar{i}$ is the image of $i$ in $\mathbb{Z} / 2 \mathbb{Z}$. In particular, $e \in \mathfrak{g}_{[1]}$.

Proposition 6.1. For any $\sigma \in \operatorname{lnv}(\mathfrak{g})$, the Satake diagrams $\operatorname{Sat}(\check{\sigma})$ and $\operatorname{Sat}(\sigma \check{\sigma})$ have IBN. Moreover, the set of black nodes of either of them is contained in the set of zeros of $\mathcal{D}(e)$.

Proof. This readily follows from Proposition 3.9, since $e \in \mathfrak{g}_{1}^{(\check{\sigma})}$ and $e \in \mathfrak{g}_{1}^{(\sigma \check{\sigma})}$.

The Satake diagrams with IBN can be extracted from [24, Table 4] or tables in [20]. One can also use information on generic stabilisers for $\mathfrak{g}_{0}$-modules $\mathfrak{g}_{1}$ (cf. the proof of Prop. 3.9). For instance, let $\sigma_{n, m}$ be an involution of $\mathfrak{g}=\mathfrak{s o}_{n+m}$ such that $\mathfrak{g}^{\sigma_{n, m}}=\mathfrak{s o}_{n} \dot{+} \mathfrak{s o}_{m}$. The $\mathfrak{g}_{0}$-module $\mathfrak{g}_{1}$ is isomorphic to $\mathbb{C}^{n} \otimes \mathbb{C}^{m}$ and a generic stabiliser is $\mathfrak{s o}|n-m|$. Therefore, $\operatorname{Sat}\left(\sigma_{n, m}\right)$ has only IBN if and only if $|n-m| \leqslant 4$. Another example is that the Satake diagram of the PI-involution $\vartheta$ has no black nodes at all (but has some arrows if $\mathfrak{g}=$ $\mathbf{A}_{n}, \mathbf{D}_{2 n+1}$, or $\left.\mathbf{E}_{6}\right)$.

Recall from [4] that a subalgebra $\mathfrak{k}$ of $\mathfrak{g}$ is said to be regular, if it is normalised by a Cartan subalgebra. For $\sigma$ inner, $\mathfrak{g}^{\sigma}=\mathfrak{g}_{0}$ is a regular reductive subalgebra. Therefore, if $e \in \mathfrak{g}_{0, \text { reg }} \cap \mathcal{N}$, then $\left[\mathfrak{g}_{0}, \mathfrak{g}_{0}\right]$ is a "minimal including regular subalgebra" (= MIRS) for $G \cdot e$ (in the terminology of [4]). For $\mathfrak{g}$ exceptional and $e \in \mathcal{N}$, Dynkin explicitly determined all MIRS for $G \cdot e$, see Tables 16-20 in [4]. (A few inaccuracies has been corrected in [5]). Therefore, it is rather easy to determine $G \cdot e$ and $\mathcal{D}(e)$ for the inner involutions.

Example 6.2. For $\left(\mathfrak{g}, \mathfrak{g}_{0}\right)=\left(\mathbf{E}_{7}, \mathbf{D}_{6}+\mathbf{A}_{1}\right)$ and $e \in \mathfrak{g}_{0, \text { reg }}$, the subalgebra $\mathbf{D}_{6} \dot{+} \mathbf{A}_{1}$ is a MIRS of $\mathfrak{g}$ for $G \cdot e$. Then browsing Table 19 in [4] or Table 5 in [5] one finds $\mathcal{D}(e)$. This orbit is 
denoted nowadays as $\mathrm{E}_{7}\left(a_{3}\right)$ and $\mathcal{D}(e)=2$-2)-20-2) Since the Satake diagram for $\left(\mathfrak{g}, \mathfrak{g}_{0}\right)$ is

By [10, Theorem 6], there is a unique maximal nilpotent orbit $G \cdot e^{\prime}$ such that $G \cdot e^{\prime} \cap \mathfrak{g}_{1} \neq$ $\varnothing$. Here $\mathcal{D}\left(e^{\prime}\right)$ is obtained from Sat $(\sigma)$ as follows. Set $\alpha\left(h^{\prime}\right)=2$ (resp. $\alpha\left(h^{\prime}\right)=0$ ) if $\alpha \in \Pi$ represents a white (resp. black) node of Sat $(\sigma)$.

It is not hard to determine the conjugacy class of both $\Upsilon(\sigma)=\check{\sigma}$ and $\sigma \check{\sigma}$ for all $\sigma \in \operatorname{lnv}(\mathfrak{g})$, excluding the inner involutions of $\mathfrak{s l}_{2 n+1}$. First, one describes the structure of $\mathfrak{g}_{0}$ and $\mathfrak{g}_{1}$ as $\langle e, h, f\rangle$-module. For the classical $\mathfrak{g}$, one can use the partition $\boldsymbol{\lambda}(e)$; while if $\mathfrak{g}$ is exceptional, then tables of [11] are helpful. This allows us easily to compute $\operatorname{dim} \mathfrak{g}_{\{0\}}-\operatorname{dim} \mathfrak{g}_{\{1\}}$ and $\operatorname{dim} \mathfrak{g}_{[0]}-\operatorname{dim} \mathfrak{g}_{[1]}$. And the conjugacy class of $\tilde{\sigma}$ such that $\operatorname{Sat}(\tilde{\sigma})$ has only IBN is uniquely determined by $\operatorname{dim} \mathfrak{g}^{\tilde{\sigma}}$, see below.

Lemma 6.3. If $\mathfrak{g}_{0}=\sum_{k \geqslant 0} m_{0, k} \mathrm{R}_{2 k}$ and $\mathfrak{g}_{1}=\sum_{k \geqslant 0} m_{1, k} \mathrm{R}_{2 k}$ as $\langle e, h, f\rangle$-modules, then

$$
\begin{gathered}
\operatorname{dim} \mathfrak{g}_{\{0\}}-\operatorname{dim} \mathfrak{g}_{\{1\}}=\sum_{k \geqslant 0}(-1)^{k} m_{0, k}+\sum_{k \geqslant 0}(-1)^{k} m_{1, k} \quad(\text { for } \check{\sigma}) ; \\
\operatorname{dim} \mathfrak{g}_{[0]}-\operatorname{dim} \mathfrak{g}_{[1]}=\sum_{k \geqslant 0}(-1)^{k} m_{0, k}+\sum_{k \geqslant 0}(-1)^{k+1} m_{1, k} \quad(\text { for } \sigma \check{\sigma}) .
\end{gathered}
$$

Proof. It follows from (6.1) and (6.2) that $\mathfrak{g}_{0} \cap \mathfrak{g}_{\{0\}}=\mathfrak{g}_{0} \cap \mathfrak{g}_{[0]}$ and the $h$-eigenvalues here are multiples of 4 . Therefore, if $\mathrm{R}_{2 k} \subset \mathfrak{g}_{0}$, then

$$
\operatorname{dim}\left(\mathrm{R}_{2 k} \cap \mathfrak{g}_{\{0\}}\right)=\operatorname{dim}\left(\mathrm{R}_{2 k} \cap \mathfrak{g}_{[0]}\right)=\left\{\begin{array}{ll}
k, & \text { if } k \text { is odd } \\
k+1, & \text { if } k \text { is even }
\end{array} .\right.
$$

On the other hand, $\mathfrak{g}_{1} \cap \mathfrak{g}_{\{0\}}=\mathfrak{g}_{1} \cap \mathfrak{g}_{[1]}$. Therefore if $\mathrm{R}_{2 k} \subset \mathfrak{g}_{1}$, then

$$
\operatorname{dim}\left(\mathrm{R}_{2 k} \cap \mathfrak{g}_{\{0\}}\right)=\operatorname{dim}\left(\mathrm{R}_{2 k} \cap \mathfrak{g}_{[1]}\right)=\left\{\begin{array}{ll}
k, & \text { if } k \text { is odd } \\
k+1, & \text { if } k \text { is even }
\end{array} .\right.
$$

This means that $\mathrm{R}_{2 k} \subset \mathfrak{g}_{0}$ contributes ' $(-1)^{k \prime}$ to both differences in the lemma, while $\mathrm{R}_{2 k} \subset \mathfrak{g}_{1}$ contributes ' $(-1)^{k \prime}$ to $(6 \cdot 3)$ and ' $(-1)^{k+1 \text { ' }}$ to $(6 \cdot 4)$.

Remark 6.4. For classical Lie algebras, the structure of $\mathfrak{g}_{0}$ and $\mathfrak{g}_{1}$ as $\langle e, h, f\rangle$-modules usually involves terms of the form $\mathrm{R}_{m} \otimes \mathrm{R}_{m+2 k}$ or $\mathcal{S}^{2} \mathrm{R}_{m}$ or $\wedge^{2} \mathrm{R}_{m}$. Then it is easy to compute the contribution of the whole such aggregates to the differences in Lemma 6.3, see Example 6.6 below.

Lemma 6.5. If the Satake diagram of $\sigma \in \operatorname{lnv}(\mathfrak{g})$ has only IBN, then

$$
\operatorname{dim} \mathfrak{g}_{1}-\operatorname{dim} \mathfrak{g}_{0}=\operatorname{rk}(\mathfrak{g})-2 \cdot \#\{\text { arrows }\}-4 \cdot \#\{\text { black nodes }\} .
$$

Furthermore, for given $\mathfrak{g}$, this quantity distinguishes different Satake diagrams with IBN. 
Proof. For $x \in \mathfrak{g}_{1}$, the value $\operatorname{dim} \mathfrak{g}_{1}^{x}-\operatorname{dim} \mathfrak{g}_{0}^{x}$ does not depend on $x$ [10, Proposition 5]. Hence the value for $x=0$, which we need, can be computed via a generic semisimple $x \in \mathfrak{g}_{1}$. Then $\mathfrak{g}^{x}$ is a Levi subalgebra of $\mathfrak{g}$ whose semisimple part corresponds to the subdiagram of black nodes of $\operatorname{Sat}(\sigma)$ and $\mathfrak{g}_{1}^{x}$ is Cartan subspace of $\mathfrak{g}_{1}$. Therefore

$$
\operatorname{dim} \mathfrak{g}_{1}^{x}=\#\{\text { white nodes }\}-\#\{\text { arrows }\}=\operatorname{rk}(\mathfrak{g})-\#\{\text { black nodes }\}-\#\{\text { arrows }\}
$$

and $\operatorname{dim} \mathfrak{g}_{0}^{x}=\operatorname{dim}\left[\mathfrak{g}^{x}, \mathfrak{g}^{x}\right]+\#\{$ arrows $\}$. Finally, if Sat $(\sigma)$ has only $I B N$, then $\left[\mathfrak{g}^{x}, \mathfrak{g}^{x}\right] \simeq\left(\mathfrak{s l}_{2}\right)^{k}$, where $k=\#\{$ black nodes $\}$.

Now, a straightforward verification shows that, for a fixed $\mathfrak{g}$ and the Satake diagrams with only $I B N$, the quantities $\#\{$ arrows $\}+2 \cdot \#\{$ black nodes $\}$ are all different.

Example 6.6. Let $\mathfrak{g}=\mathfrak{s o}_{2 n}=\mathfrak{s o}(\mathbb{V})$ and let $\sigma \in \operatorname{Inv}\left(\mathfrak{s o}_{2 n}\right)$ be such that $\mathfrak{g}^{\sigma}=\mathfrak{g l}_{n}$. Then $\sigma$ is inner, $\operatorname{dim} \mathfrak{g}_{0}-\operatorname{dim} \mathfrak{g}_{1}=n$, and $\operatorname{Sat}(\sigma)$ is

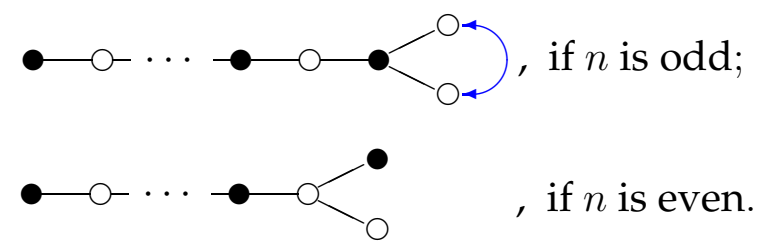

These different Satake diagrams suggest that $\check{\sigma}$ and $\sigma \check{\sigma}$ might also depend on the parity of $n$. Indeed, if $e \in \mathfrak{g}_{0, \text { reg }} \cap \mathcal{N}$, then $\boldsymbol{\lambda}(e)=(n, n)$ and $\mathbb{V}=\mathrm{R}_{n-1} \oplus \mathrm{R}_{n-1}$. Therefore $\mathfrak{g}_{0}=\mathrm{R}_{n-1} \otimes \mathrm{R}_{n-1}$ and $\mathfrak{g}_{1}=2 \cdot \wedge^{2} \mathrm{R}_{n-1}$. The number of simple $\mathfrak{s l}_{2}$-modules in $\mathfrak{g}_{0}$ (resp. $\mathfrak{g}_{1}$ ) equals $n$ (resp. $2 \cdot[n / 2]$ ).

(i) Suppose that $n=2 m+1$. Using Lemma 6.3 and the Clebsch-Gordan formulae, we see that the contribution of all $\mathfrak{s l}_{2}$-modules in $\mathfrak{g}_{0}$ (resp. $\mathfrak{g}_{1}$ ) to the difference dim $\mathfrak{g}_{\{0\}}-$ $\operatorname{dim} \mathfrak{g}_{\{1\}}$ equals 1 (resp. $\left.-2 m=-2 \cdot[n / 2]\right)$. Hence $\operatorname{dim} \mathfrak{g}_{\{0\}}-\operatorname{dim} \mathfrak{g}_{\{1\}}=1-2 m=-(\mathrm{rkg}-2)$. By Lemma 6.5, this can only happen if Sat $(\check{\sigma})$ has one arrow and no black nodes. Hence $\check{\sigma}=\vartheta$ is a PI-involution with $\mathfrak{g}^{\vartheta} \simeq \mathfrak{s o}_{2 m+2}+\mathfrak{s o}_{2 m}=\mathfrak{s o}_{n+1}+\mathfrak{s o}_{n-1}$.

For $\sigma \check{\sigma}$, the contribution from the $\mathfrak{s l}_{2}$-modules in $\mathfrak{g}_{1}$ is counted with the opposite sign, i.e., $\operatorname{dim} \mathfrak{g}_{[0]}-\operatorname{dim} \mathfrak{g}_{[1]}=1+2 m=\mathrm{rk}(\mathfrak{g})$. This means that $\sigma$ and $\sigma \check{\sigma}$ are $G$-conjugate.

(ii) Suppose that $n=2 \mathrm{~m}$. Then the $\mathfrak{s l}_{2}$-modules in $\mathfrak{g}_{0}$ make zero contribution to either of the differences, while the contribution from $\mathfrak{s l}_{2}$-modules in $\mathfrak{g}_{1}$ to (6.3) and (6.4) equals $n$ and $-n$, respectively. Hence $\operatorname{dim} \mathfrak{g}_{\{0\}}-\operatorname{dim} \mathfrak{g}_{\{1\}}=n=\operatorname{rkg}$ and $\operatorname{dim} \mathfrak{g}_{[0]}-\operatorname{dim} \mathfrak{g}_{[1]}=$ $-n=-\mathrm{rk}(\mathfrak{g})$. This means that $\check{\sigma}$ and $\sigma$ are conjugate, while $\sigma \check{\sigma}$ is of maximal rank, i.e., $\mathfrak{g}^{\sigma \check{\sigma}} \simeq \mathfrak{s o}_{n} \dot{+} \mathfrak{s o}_{n}$.

Example 6.7. For the PI-involution $\vartheta$ and $\mathfrak{g} \neq \mathbf{A}_{2 n}$, we have

- $\Upsilon(\vartheta)=\check{\vartheta} \sim \vartheta$ unless $\mathfrak{g} \in\left\{\mathbf{A}_{4 n+1}, \mathbf{B}_{4 n+1}, \mathbf{C}_{2 n+1}, \mathbf{D}_{4 n+2}, \mathbf{E}_{7}\right\}$;

- If $\mathfrak{g}=\mathbf{A}_{4 n+1}$, then $\mathfrak{g}^{\vartheta}=\mathbf{A}_{2 n} \dot{+} \mathbf{A}_{2 n} \dot{+} \mathfrak{t}_{1}$ and $\mathfrak{g}^{\Upsilon(\vartheta)}=\mathbf{A}_{2 n+1}+\mathbf{A}_{2 n-1} \dot{+} \mathfrak{t}_{1} ;$

- If $\mathfrak{g}=\mathbf{B}_{4 n+1}$, then $\mathfrak{g}^{\vartheta}=\mathbf{B}_{2 n}+\mathbf{D}_{2 n+1}$ and $\mathfrak{g}^{\Upsilon(\vartheta)}=\mathbf{B}_{2 n+1}+\mathbf{D}_{2 n}$; 
- If $\mathfrak{g}=\mathbf{C}_{2 n+1}$, then $\mathfrak{g}^{\vartheta}=\mathfrak{g l}_{2 n+1}$ and $\mathfrak{g}^{\Upsilon(\vartheta)}=\mathbf{C}_{n+1}+\mathbf{C}_{n}$;

- If $\mathfrak{g}=\mathbf{D}_{4 n+2}$, then $\mathfrak{g}^{\vartheta}=\mathbf{D}_{2 n+1}+\mathbf{D}_{2 n+1}$ and $\mathfrak{g}^{\Upsilon(\vartheta)}=\mathbf{D}_{2 n+2}+\mathbf{D}_{2 n}$;

- If $\mathfrak{g}=\mathbf{E}_{7}$, then $\mathfrak{g}^{\vartheta}=\mathbf{A}_{7}$ and $\mathfrak{g}^{\Upsilon(\vartheta)}=\mathbf{D}_{6}+\mathbf{A}_{1}$.

For computations in $\mathbf{E}_{7}$ and $\mathbf{E}_{8}$, one can use data from Example 5.5(1). Further calculations show that one always has $\Upsilon^{2}(\vartheta) \sim \vartheta$. Note however that, in general, $\Upsilon^{2}(\sigma)$ has no relation to $\sigma \check{\sigma}$.

Since $\sigma$ and $\check{\sigma}$ commute, they determine a $\mathbb{Z}_{2} \times \mathbb{Z}_{2}$-grading or quaternionic decomposition $\mathfrak{g}=\bigoplus_{i, j \in \mathbb{Z}_{2} \times \mathbb{Z}_{2}} \mathfrak{g}_{i j}$. We refer to $[6,15,16]$ for various invariant-theoretic results related to this structure. However, it is worth stressing that our construction gives an ordered triple of involutions. For, starting from $\check{\sigma}$ or $\sigma \check{\sigma}$, one usually obtains another triple and a different $\mathbb{Z}_{2} \times \mathbb{Z}_{2}$-grading.

As a complement to Theorem 5.1, we have

Theorem 6.8. Suppose that $\sigma \in \operatorname{Inv}(\mathfrak{g}), e \in \mathfrak{g}_{0} \cap \mathcal{N}$ is regular in $\mathfrak{g}_{0}$, and $\mathfrak{g}=\bigoplus_{i, j \in \mathbb{Z} \times \mathbb{Z}_{2}} \mathfrak{g}_{j}(2 i)$ is the related $(\sigma, e)$-grading. If $d_{0}(4 k+2)=d_{1}(4 k+2)$ for all $k \in \mathbb{Z}$, then $\operatorname{dim} \mathfrak{g}^{\sigma}=\operatorname{dim} \mathfrak{g}^{\sigma \sigma}$. Moreover, if $\mathfrak{g}_{0}$ is semisimple, then $\sigma$ and $\sigma \check{\sigma}$ are conjugate involutions.

Proof. By Proposition 3.6, e remains even in $\mathfrak{g}$, which justifies our notation for the mixed grading. Recall that

$$
\mathfrak{g}^{\sigma}=\mathfrak{g}_{0}=\bigoplus_{i \in \mathbb{Z}} \mathfrak{g}_{0}(2 i), \mathfrak{g}^{\check{\sigma}}=\bigoplus_{i \in \mathbb{Z}} \mathfrak{g}(4 i) \text {, and } \mathfrak{g}^{\sigma \check{\sigma}}=\bigoplus_{i \in \mathbb{Z}} \mathfrak{g}_{\bar{i}}(2 i),
$$

where $\bar{i}$ is the image of $i$ in $\mathbb{Z} / 2 \mathbb{Z}$. Hence $\operatorname{dim} \mathfrak{g}^{\sigma}-\operatorname{dim} \mathfrak{g}^{\sigma \check{\sigma}}=\sum_{k \in \mathbb{Z}}\left(d_{0}(4 k+2)-d_{1}(4 k+2)\right)$, which proves the first assertion.

If $\mathfrak{g}_{0}$ is semisimple and $e \in \mathfrak{g}_{0, \text { reg }}$, then $d_{0}(0)=d_{0}(2)=\mathrm{rk}\left(\mathfrak{g}_{0}\right)$. Hence $d_{0}(0)=d_{1}(2)$. It then follows from Corollary 3.10 that $\operatorname{Sat}(\sigma)$ has $I B N$. Since $\operatorname{dim} \mathfrak{g}^{\sigma}=\operatorname{dim} \mathfrak{g}^{\sigma \sigma}$ and both involutions have only IBN in their Satake diagrams, they must be $G$-conjugate.

Remark 6.9. Actually, the semisimplicity of $\mathfrak{g}_{0}$ is not necessary, as long as we know that Sat $(\sigma)$ has only $I B N$. The equalities $d_{0}(4 k+2)=d_{1}(4 k+2)$ are often related to the fact that $G \cdot e$ is divisible and $G \cdot e^{\langle 2\rangle} \cap \mathfrak{g}_{1}(4) \neq \varnothing$, cf. the proof of Theorem 5.1. Hence Theorem 6.8 applies if $d_{0}(0)=d_{1}(4)$. But there are many other cases, where $\sigma \sim \sigma \check{\sigma}$, see e.g. Example 6.6 with $n$ odd.

Example 6.10. It can happen that $\sigma=\vartheta_{\max }$ with $\mathfrak{g}^{\sigma}$ semisimple, but the involutions $\sigma$ and $\sigma \check{\sigma}$ are not $G$-conjugate. Suppose that $\mathfrak{g}=\mathbf{B}_{4 n+2}$ and $\sigma$ is of maximal rank, which is also a PI-involution here. Then $\mathfrak{g}_{0}=\mathfrak{g}^{\sigma} \simeq \mathfrak{g}^{\check{\sigma}} \simeq \mathbf{B}_{2 n+1}+\mathbf{D}_{2 n+1}$, but $\mathfrak{g}^{\sigma \check{\sigma}}=\mathbf{B}_{2 n} \dot{+} \mathbf{D}_{2 n+2}$. The reason is that $G \cdot e$ is not divisible for $e \in \mathfrak{g}_{0, \text { reg }}$ and the equality $d_{0}(4 k+2)=d_{1}(4 k+2)$ fails exactly for $k=n,-n-1$. This leads to the relation $\operatorname{dim} \mathfrak{g}^{\sigma \check{\sigma}}-\operatorname{dim} \mathfrak{g}^{\sigma}=2$.

Example 6.11. There is an interesting case in which three involutions $\sigma, \check{\sigma}$, and $\sigma \check{\sigma}$ are pairwise non-conjugate. Let $\sigma \in \operatorname{Inv}(\mathfrak{g})$ be a diagram involution for $\mathfrak{g}=\mathbf{A}_{2 n-1}, \mathbf{D}_{n}$, and $\mathbf{E}_{6}$. 
(See [7, Ch. 8] for diagram automorphisms of simple Lie algebras.) Then $\sigma$ is outer, $\mathfrak{g}^{\sigma}$ is simple, and if $e \in \mathfrak{g}_{0, \text { reg }}$, then $e \in \mathfrak{g}_{\text {reg }}$. By the above "first properties", $\check{\sigma}$ is inner and $\sigma \check{\sigma}$ is outer. Since $e \in \mathfrak{g}_{1}^{(\check{\sigma})}$, this implies that $\check{\sigma}=\vartheta$ is a PI-involution. Furthermore, $\sigma \check{\sigma}$ is the unique, up to $G$-conjugacy, outer involution in the connected component of $\operatorname{Aut}(\mathfrak{g})$ containing $\sigma$ that has the property that $e \in \mathfrak{g}_{1}^{(\sigma \check{\sigma})}$, i.e., the $(-1)$-eigenspace of the outer involution $\sigma \check{\sigma}$ contains a regular nilpotent element of $\mathfrak{g}$. If $\vartheta$ is not of maximal rank, then $\sigma \check{\sigma}$ appears to be of maximal rank. Below is the table with fixed point subalgebras for this triple of involutions, where $\sigma \check{\sigma} \nsim \vartheta_{\max }$ only for $\mathfrak{g}=\mathbf{D}_{2 n}$.

TABLE 3. Diagram involutions $\sigma$ and related triples

\begin{tabular}{l|lll|}
$\mathfrak{g}$ & $\mathfrak{g}^{\sigma}$ & $\mathfrak{g}^{\check{\sigma}}=\mathfrak{g}^{\vartheta}$ & $\mathfrak{g}^{\sigma \check{\sigma}}$ \\
\hline \hline $\mathbf{A}_{2 n-1}$ & $\mathbf{C}_{n}$ & $\mathbf{A}_{n-1} \dot{+} \mathbf{A}_{n-1} \dot{+} \mathfrak{t}_{1}$ & $\mathbf{D}_{n}$ \\
$\mathbf{D}_{2 n}$ & $\mathbf{B}_{2 n-1}$ & $\mathbf{D}_{n} \dot{+} \mathbf{D}_{n}$ & $\mathbf{B}_{n} \dot{+} \mathbf{B}_{n-1}$ \\
$\mathbf{D}_{2 n+1}$ & $\mathbf{B}_{2 n}$ & $\mathbf{D}_{n} \dot{+} \mathbf{D}_{n+1}$ & $\mathbf{B}_{n} \dot{+} \mathbf{B}_{n}$ \\
$\mathbf{E}_{6}$ & $\mathbf{F}_{4}$ & $\mathbf{A}_{5} \dot{+} \mathbf{A}_{1}$ & $\mathbf{C}_{4}$ \\
\hline
\end{tabular}

For $\mathfrak{g}=\mathbf{A}_{2 n}$, the diagram involution $\sigma$ is of maximal rank. Here this procedure provides $G$-conjugate involutions $\sigma$ and $\sigma \check{\sigma}$.

\section{REFERENCES}

[1] Л.В. Антонян. О классификации однородных элементов $\mathbb{Z}_{2}$-градуированных полупростых алгебр Ли, Вестник Моск. Ун-та, Сер. Матем. Мех., № 2 (1982), 29-34 (Russian). English translation: L.V. ANTONYAN. On classification of homogeneous elements of $\mathbb{Z}_{2}$-graded semisimple Lie algebras, Moscow Univ. Math. Bulletin, 37 (1982), № 2, 36-43.

[2] A. Broer. Normality of some nilpotent varieties and cohomology of line bundles on the cotangent bundle of the flag variety, In: J.-L. Brylinski, R. Brylinski, V. Guillemin and V. Kac (Eds.) "Lie Theory and Geometry. In honor of Bertram Kostant", Progr. Math. 123, 1-19, Birkhäuser Boston, 1994.

[3] D.H. Collingwood and W. MCGOvern. "Nilpotent orbits in semisimple Lie algebras", New York: Van Nostrand Reinhold, 1993.

[4] Е.Б. Дынкин. Полупростые подалгебры полупростых алгебр Ли, Матем. Сборник, т.30, № 2 (1952), 349-462 (Russian). English translation: E.B. DYNKIN. Semisimple subalgebras of semisimple Lie algebras, Amer. Math. Soc. Transl., II Ser., 6 (1957), 111-244.

[5] A.G. ElashViLI. The centralizers of nilpotent elements in semisimple Lie algebras, Trudy Razmadze Matem. Inst. (Tbilisi) 46 (1975), 109-132 (Russian). (MR0393148)

[6] A. HELMINCK and G. SCHWARZ. Orbits and invariants associated with a pair of commuting involutions, Duke Math. J., 106 (2001), 237-279.

[7] V.G. KAC. "Infinite-dimensional Lie algebras”, 3rd edition. Cambridge University Press, Cambridge, 1990. xxii+400 pp.

[8] G. KEMPF. On the collapsing of homogeneous bundles, Invent. Math., 37, no. 3 (1976), 229-239.

[9] B. Kostant. The principal three-dimensional subgroup and the Betti numbers of a complex simple Lie group, Amer. J. Math., 81 (1959), 973-1032. 
[10] B. Kostant and S. RaLlis. Orbits and representations associated with symmetric spaces, Amer. J. Math., 93 (1971), 753-809.

[11] R. LAWTHER. Jordan block sizes of unipotent elements in exceptional algebraic groups, Comm. Alg., 23 (1995), 4125-4156.

[12] D. LunA. Slices ètales, Bull. Soc. Math. France, Memoire 33 (1973), 81-105.

[13] D. PAnYushev. On invariant theory of $\theta$-groups, J. Algebra, 283 (2005), 655-670.

[14] D. PAnYushev. On divisible weighted Dynkin diagrams and reachable elements, Transformation Groups, 15, no. 4 (2010), 983-999.

[15] D. PANYUSHEV. Commuting involutions and degenerations of isotropy representations, Transformation Groups, 18, no. 2 (2013), 507-537.

[16] D. PAnyushev. Commuting involutions of Lie algebras, commuting varieties, and simple Jordan algebras, Algebra Number Theory, 7, no. 6 (2013), 1505-1534.

[17] D. PANYUSHEV and O. YAKIMOVA. On maximal commutative subalgebras of Poisson algebras associated with involutions of semisimple Lie algebras, Bull. Sci. Math., 138, no. 6 (2014), 705-720.

[18] E. SOMmers. B-stable ideals in the nilradical of a Borel subalgebra, Canad. Math. Bull., 48 (2005), 460-472.

[19] T.A. SpRINGER. "Invariant theory". Lecture Notes Math., vol. 585. Springer-Verlag, Berlin-New York, 1977. iv+112 pp.

[20] T.A. Springer. The classification of involutions of simple algebraic groups, J. Fac. Sci. Univ. Tokyo Sect. IA Math. 34 (1987), 655-670.

[21] T.A. Springer and R. Steinberg. Conjugacy Classes, In: "Seminar on algebraic and related finite groups", Lecture Notes Math., Berlin: Springer, 131 (1970), 167-266.

[22] Э.Б. ВинБеРГ. Группа Вейля градуированной алгебры Ли, Изв. АН СССР. Сер. Матем. 40, № 3 (1976), 488-526 (Russian). English translation: E.B. VINBERG. The Weyl group of a graded Lie algebra, Math. USSR-Izv. 10 (1976), 463-495.

[23] Э.Б. Виньерг. Классификация однородных нильпотентных элементов полупростой градуированной алгебры Ли, В сб.: ”Труды семинара по вект. и тенз. анализу", т. 19, стр. 155-177. Москва: МГУ 1979 (Russian). English translation: E.B. VINBERG. Classification of homogeneous nilpotent elements of a semisimple graded Lie algebra, Selecta Math. Sov., 6 (1987), 15-35.

[24] Э.Б. ВинБеРГ, В.В. ГорьАцеВИч, А.Л. ОНИщик. “Группы и алгебры Ли - 3”, Соврем. пробл. математики. Фундам. направл., т. 41. Москва: ВИНИТИ 1990 (Russian). English translation: V.V. Gorbatsevich, A.L. Onishchik and E.B. Vinberg. "Lie Groups and Lie Algebras" III (Encyclopaedia Math. Sci., vol. 41) Berlin: Springer 1994.

INSTITUTE FOR INFORMATION TRANSMISSION PROBLEMS OF THE R.A.S., BOLSHOI KARETNYI PER. 19, MOSCOW 127051, RusSiA

Email address: panyushev@iitp.ru 